\title{
Synthèse des dix premières années de suivi hydrobiologique du Rhône au niveau de la centrale nucléaire de Saint-Alban (France)
}

\section{Ten years of hydrobiological monitoring of the Rhone River near Saint-Alban nuclear power plant (France)}

\author{
Jean-François Fruget ${ }^{(1,2)}$, Michel Centofanti ${ }^{(1,2)}$, \\ Jeanne Dessaix ${ }^{(1,2)}$, Jean-Michel Olivier ${ }^{(2)}$, \\ Jean-Claude Druart ${ }^{(3)}$ \& Pierre-Jean Martinez ${ }^{(4)}$ \\ (1) ARALEPBP, Biologie Animale, Université Lyon I, Bât. 403, 69622 Villeurbanne cedex, \\ France. \\ (2) ESA CNRS 5023 "Ecologie des Eaux Douces et des Grands Fleuves ", Université Lyon I, \\ 69622 Villeurbanne cedex France. \\ (3) INRA, Institut de Limnologie, Station d'Hydrobiologie lacustre, BP 511, 74203 Thonon-les- \\ Bains cedex, France. \\ (4) GREBE, 23 rue Saint-Michel, 69007 Lyon, France.
}

Résumé. - Le bilan des dix premières années de suivi de l'influence du rejet d'eau de refroidissement de la centrale nucléaire de Saint-Alban, implantée sur le Rhône environ $50 \mathrm{~km}$ à l'aval de Lyon, a permis d'établir que les caractéristiques physiques, chimiques et biologiques du Rhône-Moyen sont issues, en premier lieu, de l'anthropisation du fleuve et de sa vallée. D'un point de vue biologique, le système a dérivé vers un type potamique lent, composé d'espèces ubiquistes et résistantes et de quelques espèces lotiques plus sensibles mais relictuelles. D'un point de vue physico-chimique, ses grandes dimensions et l'inertie que cela confère à la masse d'eau vis-à-vis des apports extérieurs permettent au milieu d'assimiler des charges minérale et organique importantes. L'influence du rejet d'eau échauffée se manifeste par une augmentation de la température de l'eau à l'aval du site, voire dans la partie court-circuitée. Les effets sur la faune et la flore sont toutefois modérés, ou non significatifs, probablement masqués par les modifications antérieures à l'implantation de la centrale, dues à l'anthropisation contemporaine du milieu (régulation et canalisation, rejets domestiques et industriels divers). L'hydrologie et la température apparaissent comme les événements majeurs régissant la dynamique physico-chimique et biologique du secteur (variations inter-annuelles des paramètres physico-chimiques, de la richesse, de la diversité et de l'abondance des différentes espèces végétales et animales). La saisonnalité des facteurs thermiques et hydrologiques et les conditions hydroclimatiques sont les principaux facteurs de contrôle. II apparait ainsi que l'étude de l'évolution des hydrosystèmes fluviaux 
et de la dynamique de leurs peuplements impliquent une continuité d'observation et de récolte de données que seules permettent des études à long terme, nécessitant la mise en place de véritables observatoires écologiques du milieu naturel.

Mots-clés - Suivi à long terme, fleuve aménagé, Rhône, physico-chimie, périphyton, phytoplancton, macrophytes, invertébrés benthiques, ichtyofaune.

\begin{abstract}
The assessment of the first ten years of monitoring of the discharge of cooling water from the Saint-Alban nuclear power plant, built on the Rhône River about $50 \mathrm{~km}$ downstream from the city of Lyon, shows that from the biological viewpoint, the river has shifted from a lotic system toward a slow-flowing potamic type, with ubiquitous resistant species plus a few more lotic and sensitive relict species. In physico-chemical terms, its large size and the inertia that this confers on the water mass in relation to external influences allows this environment to assimilate large quantities of inorganic and organic inputs. The increase of the water temperature in the reservoir downstream from the power plant, or in the by-passed section, shows the influence of the warm water discharge, but the biological effects on the fauna or the flora are moderate, or even insignificant. They are masked by previous man-made alterations that took place before the power plant was operated, such as regulation works and domestic and industrial inputs. The seasonality of thermal and hydrological factors and the hydroclimatic conditions are the main controlling factors. It therefore appears that the study of long-term changes in river systems including the dynamics of their animal and plant communities requires continuous observations and data collections that only long-term studies can provide, implying the setting up of ecological monitoring programmes for the natural environment.
\end{abstract}

Key words - Long-term survey, regulated river, Rhône River, physico-chemistry, periphyton, phytoplankton, macrophytes, macroinvertebrates, fish fauna.

\section{INTRODUCTION}

La plupart des études menées actuellement sur les grands cours d'eau ont suivi les modifications de leur fonctionnement engendrées par l'homme; les activités humaines ayant affecté fleuves et rivières partout dans le monde (Petts et al., 1989; Dynesius \& Nilsson, 1994). Parmi ces activités perturbatrices, les rejets d'eau de refroidissement des centrales électriques (nucléaires en particulier) ont pris une place de plus en plus importante avec le développement accéléré de cette forme d'énergie durant les deux dernières décennies (Khalanski \& Gras, 1996).

Le Rhône n'échappe évidemment pas à ce phénomène. Depuis près de 200 ans l'Homme a profondément modifié le Rhône-Moyen, partie du fleuve située entre Lyon et la confluence de l'Isère: endiguements du $\mathrm{XIX}{ }^{\mathrm{e}}$ siècle destinés à protéger les riverains contre les crues et à améliorer les conditions de navigation; aménagements hydroélectriques et canalisation contemporains de la Compagnie Nationale du Rhône et utilisation de l'eau du fleuve pour le 
refroidissement des centrales thermiques classiques et nucléaires (Fruget, 1992). Parallèlement à ces modifications physiques, l'extension de villes comme Lyon, et l'existence de grands complexes pétrochimiques ont modifié la composition chimique des eaux du Rhône. L'impact de ces perturbations, à la fois physiques et chimiques, restait donc à établir. L'obligation de suivi de l'influence sur le milieu aquatique du rejet d'eau de refroidissement de la centrale électro-nucléaire de Saint-Alban - St-Maurice, implantée au début des années 80 , nous en a donnés l'occasion. Le but de ce suivi est de déterminer la structure des peuplements aquatiques rencontrés et le fonctionnement des milieux inventoriés en utilisant différents paramètres abiotiques et biotiques comme outils de mesure (descripteurs de fonctionnement au sens de Bournaud \& Amoros, 1984). De par son caractère spatio-temporel, ce suivi s'inscrit dans le contexte de l'évolution récente de ce secteur du Rhône (aménagements pour la navigation et l'hydroélectricité, rejets chimiques et thermiques). Un descripteur abiotique, la physico-chimie de l'eau, et plusieurs descripteurs biotiques, particulièrement intégrateurs, les algues du phytoplancton et du périphyton, les macrophytes aquatiques, les invertébrés benthiques et l'ichtyofaune, sont ainsi échantillonnés régulièrement. Un bilan des dix premières années de suivi a été établi (ARALEPBP, 1997). Cet article en présente une version synthétique.
L'objectif de ce bilan peut être formulé sous la forme des questions suivantes: (1) quelle est la qualité hydrobiologique actuelle de ce secteur du Rhône?; (2) des changements perceptibles se sont-ils produits depuis le milieu des années 80 ?; (3) quelle est l'influence du réchauffement artificiel des eaux à l'aval de la centrale? Chaque fois que cela a été possible, les résultats ont été confrontés à ceux de la campagne de référence 1982-83 (ARALEPBP, 1983).

\section{MATÉRIEL ET MÉTHODES}

\subsection{Secteur d'étude}

Le secteur de Péage-de-Roussillon est situé sur le Rhône-Moyen environ $50 \mathrm{~km}$ à l'aval de Lyon (fig. 1A). Ce secteur du fleuve est soumis à d'importants apports domestiques et industriels, en particulier de $\mathrm{NH}_{4}$ et $\mathrm{PO}_{4}$ issus d'industries situées à l'amont (Fruget, 1992). Un aménagement hydroélectrique à dérivation y a été implanté en 1977 par la Compagnie Nationale du Rhône (CNR) puis la centrale nucléaire de Saint-Alban, localisée sur la retenue, a été progressivement mise en service à partir de 1985. A l'état naturel, le fleuve montrait un important réseau de chenaux tressés (Michelot, 1983; Fruget \& Michelot, 1997). Les endiguements du siècle dernier, puis l'aménagement CNR ont modifié cette dynamique flu- 


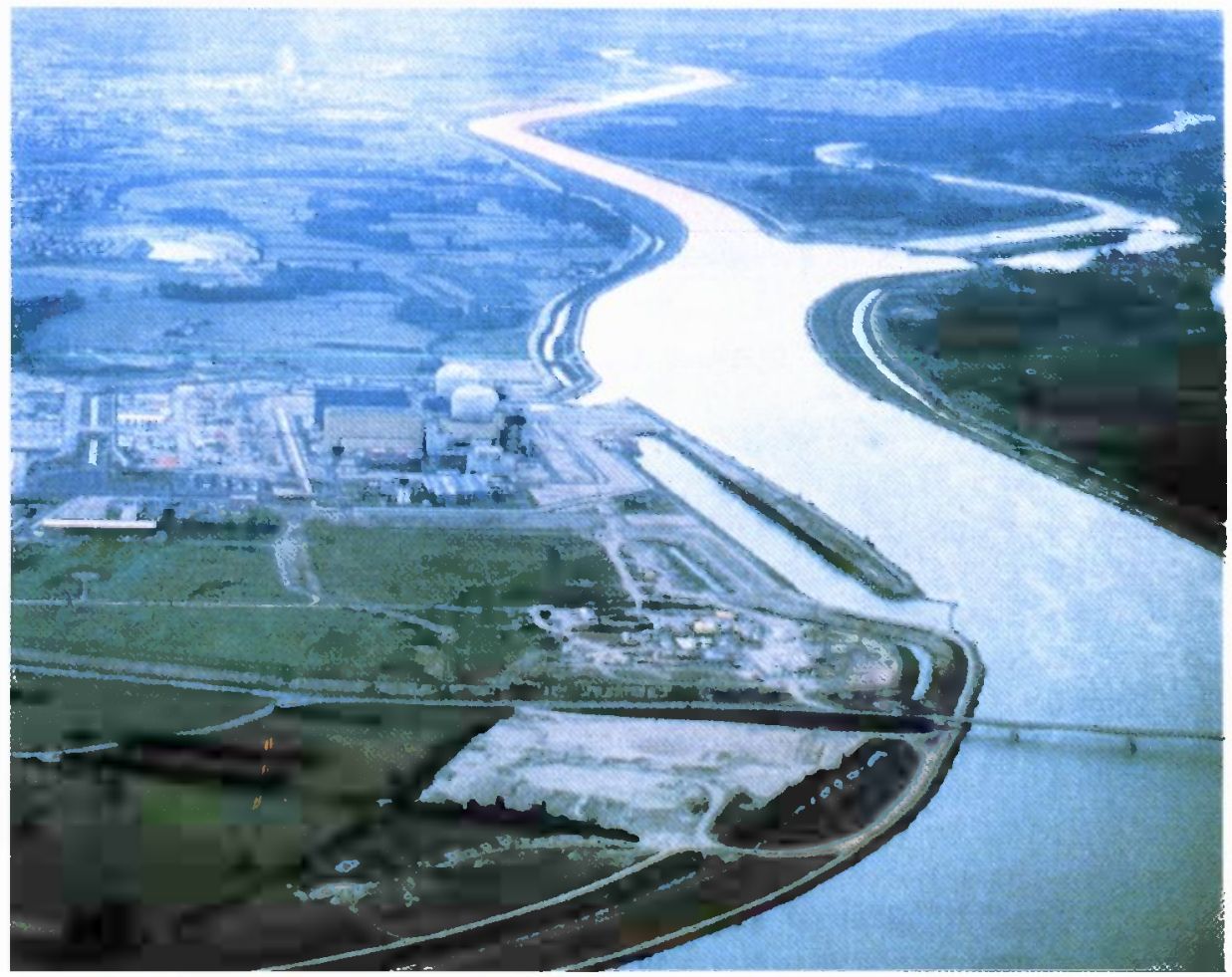

Photo 1. - Vue aérienne de la retenue de St-Pierre-de-Bceuf en direction de l'aval. Au centre, le site de la centrale de Saint-Alban et son canal d'amenée. En arrière plan, le canal de dérivation, le Rhône court-circuité et le plan d'eau de St-Pierre-de-Bceuf à l'extrême droite (Photo FRAPNA 26).

Photo 1. - Aerial view of St-Pierre-de-Bœeuf reservoir in the downstream direction. In the middle part, Saint-Alban nuclear power plant and its headrace canal. Background, the diversion canal, the by-passed section and St-Pierre-de-Bceuf pool on the right side.

viale. Toutefois, le secteur présente encore des boisements alluviaux bien conservés et plusieurs bras morts (lônes) formant un des derniers grands ensembles de milieux naturels du Rhône à l'aval de Lyon. Une grande partie de ces milieux est toutefois localisée entre le canal de dérivation et l'ancien lit du fleuve. Ce dernier est court-circuité par un barrage et ne reçoit plus qu'un très faible débit réservé en dehors des crues: 10 à $20 \mathrm{~m}^{3} / \mathrm{s}$, pour un débit moyen d'environ $1030 \mathrm{~m}^{3} / \mathrm{s}$. A côté des ensembles fonctionnels naturels définis par les différents phénomènes successionnels et géomorphologiques (lônes, Vieux-Rhône), les aménagements an- 


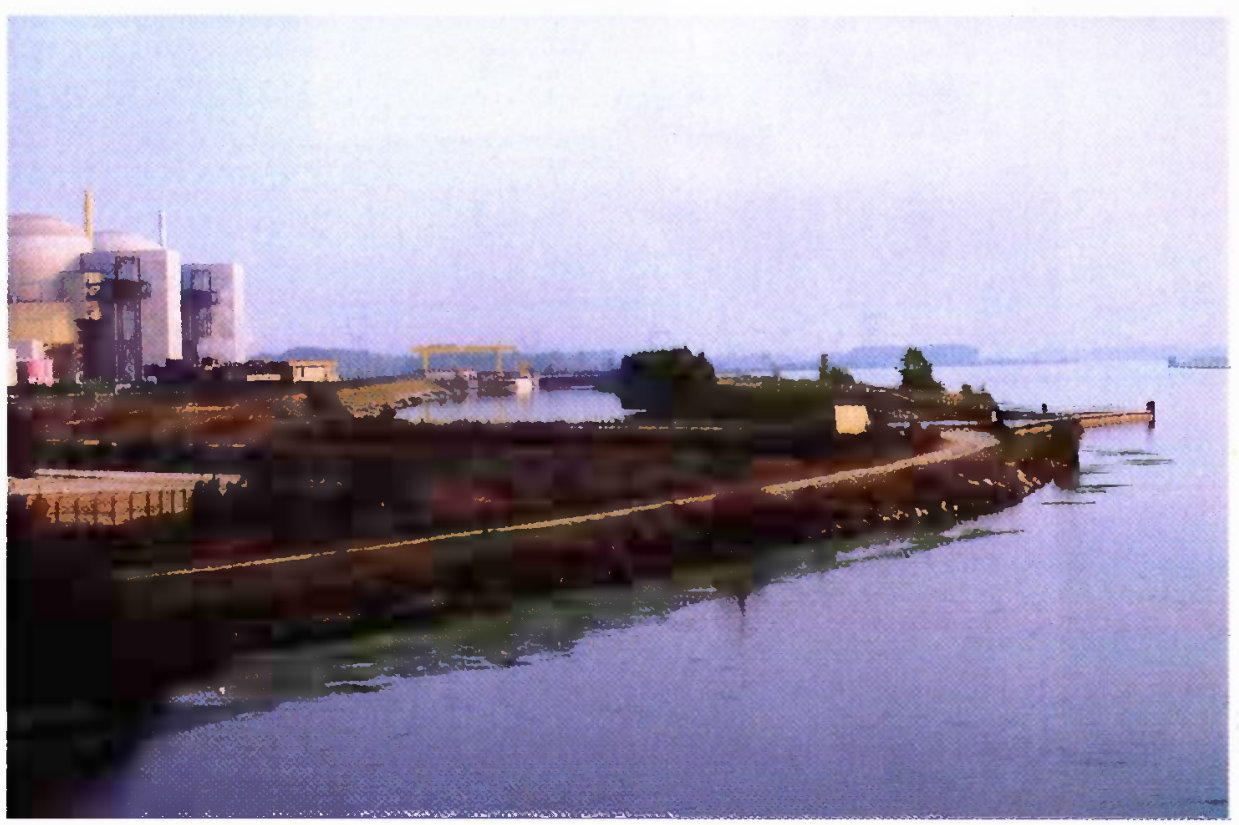

Photo 2. - Vue de la retenue et de la centrale de Saint-Alban depuis le pont de Chavanay. Le canal d'amenée de la centrale est visible au centre de la photo. Au premier plan, endiguements et zone rivulaire de la station d'échantillonnage amont (Photo J.F. Fruget).

Photo 2. - View of the reservoir and of Saint-Alban power plant from Chavanay bridge. The headrace canal of the power is visible in the middle part. Foreground, embankments and riparial zone of the upstream sampling station.

thropiques sont à l'origine d'ensembles plus ou moins artificiels tels que la retenue ou le canal de dérivation.

La centrale de St-Alban - St-Maurice est la dernière unité électro-nucléaire mise en service sur le Bas-Rhône (juillet 1985 pour la tranche 1 et août 1986 pour la tranche 2). Elle est composée de deux réacteurs de $1300 \mathrm{MW}$ électriques, refroidis en circuit ouvert par quatre pompes de $30 \mathrm{~m}^{3} / \mathrm{s}$ (soit $120 \mathrm{~m}^{3} / \mathrm{s}$ au total, lorsque l'installation fonctionne à pleine puissance). La station de pompage se situe au bout d'un canal d'amenée de $875 \mathrm{~m}$ de longueur, parallèle au Rhône. Les eaux réchauffées sont rejetées au fleuve via un canal de rejet de $400 \mathrm{~m}$ (fig. 1B).

Cinq stations d'étude ont été réparties dans les différents milieux afin d'appréhender au mieux l'incidence du rejet d'eau de refroidissement (fig. 1C) : la retenue en amont et en aval de la centrale (stations 1 et 2), le canal de dérivation (station 3 située dans le canal de fuite), le Rhône court-circuité (station 4, localisée dans un secteur endigué à courant très ralenti) et un plan d'eau corres- 
$\mathbf{A}$
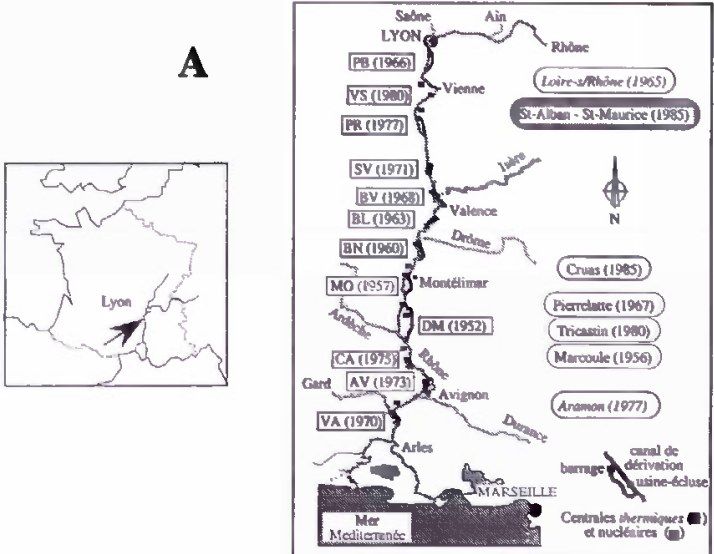

B

D
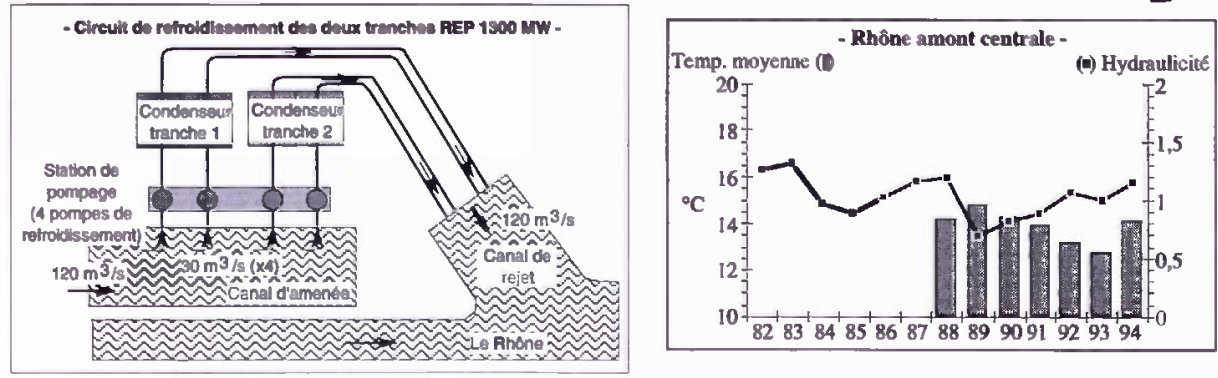

Fig. 1. - (A) Situation de la centrale nucléaire de Saint-Alban dans l'aménagement général du Bas-Rhône. (B) Système de refroidissement utilisé par les deux réacteurs REP $1300 \mathrm{MW}$ et débits mobilisés. (C) Localisation des stations de mesures du programme de surveillance hydroécologique du site de Saint-Alban. (D) Température moyenne et coefficient d'hydraulicité annuels du Rhône à l'amont de la centrale.

Fig. 1. - (A) Location of Saint-Alban nuclear power plant in the the Lower Rhône hydropower harnessing. (B) Cooling water system of Saint-Alban power plant. (C) Location of the sampling stations. (D) Average yearly temperature and relative discharge coefficient of the Rhône upstream from the power plant.

pondant à l'ancien lit court-circuité par la construction de l'évacuateur de crues du barrage (station 5 ). Les rives des stations 1, 2 et 3 sont endiguées par des enrochements de blocs tandis que celles des stations 4 et 5 sont globalement plus diversifiées et naturelles. La granulométrie du lit du chenal des stations 3 et 4 est grossière (pierres, galets, graviers) à l'opposé de celle de la retenue et du plan d'eau qui est fine (limon-vase). 


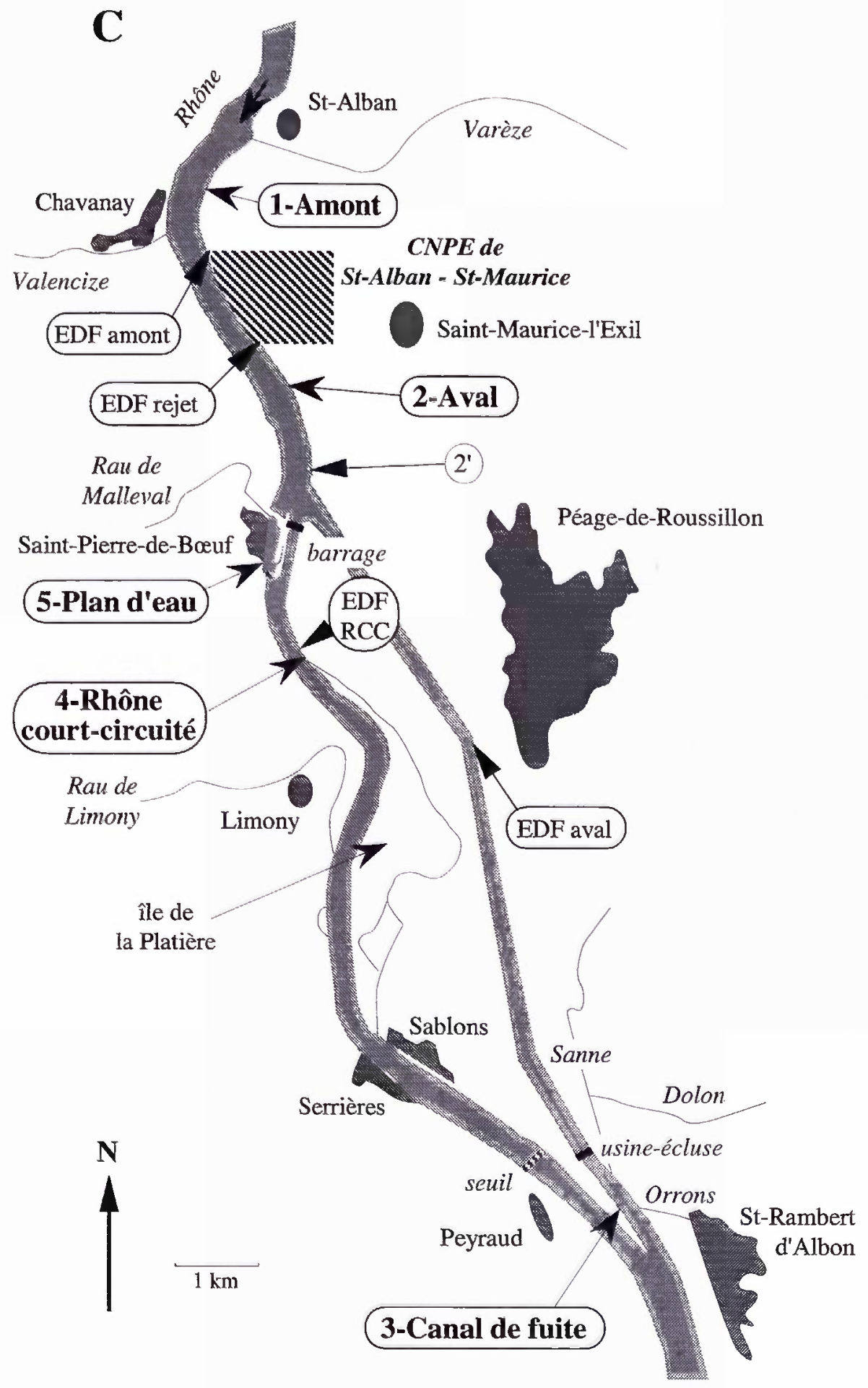



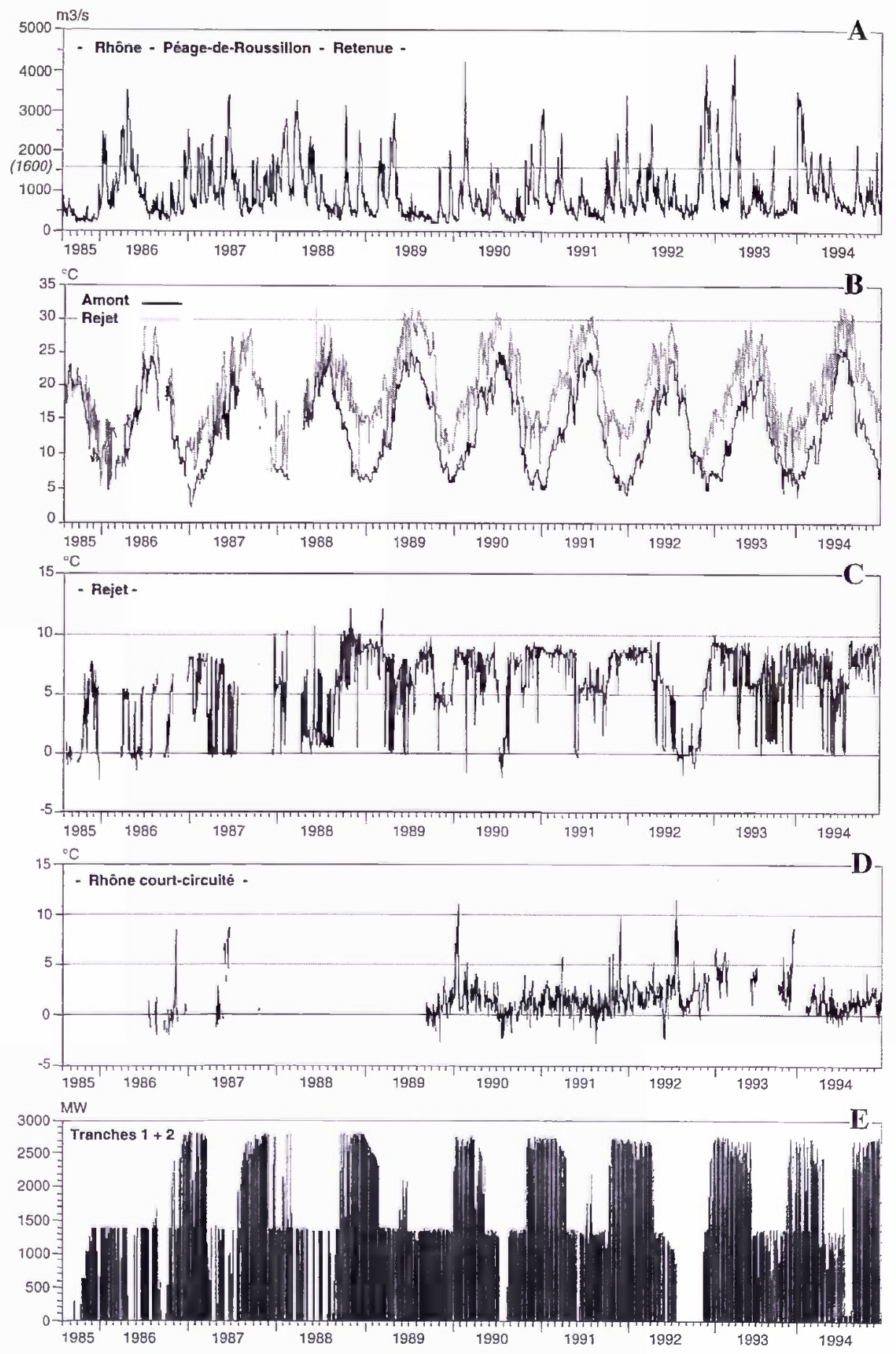
La période d'étude montre des débits particulièrement excédentaires au début des années 80 et, inversement, un déficit annuel marqué de 1989 à 1991 (fig. 1D \& 2A). Les années 82 et 83 , mais aussi 87,88 et 94 sont hydrologiquement excédentaires. A l'inverse, l'année 1989 apparait fortement déficitaire (débit moyen = $718 \mathrm{~m}^{3} / \mathrm{s}$; hydraulicité $=0,7$ ). Elle constitue une année charnière, marquant le début de trois années déficitaires. Les années 92 et 93 peuvent ensuite être qualifiées «d'hydrologiquement normales ». Cette période se termine brusquement par les importantes crues d'octobre 93 et de janvier 94. Toutefois, l'intensité de celles-ci reste inférieure à celle de la crue décennale $\left(4500 \mathrm{~m}^{3} / \mathrm{s}\right)$.

La température moyenne annuelle du Rhône à l'amont immédiat de la centrale est de $13,9^{\circ} \mathrm{C}$ pour la chronique de référence 1988-1994. Différentes périodes thermiques ont été enregistrées sur cette chronique (fig. 1D \& 2B) : 1988 à 1991 sont des années à températures moyennes annuelles «élevées» $\left(\geq 14^{\circ} \mathrm{C}\right)$; puis une diminution s'effectue de 1991 à 1993, année où la température est inférieure à $13^{\circ} \mathrm{C}$; enfin, 1994 est à nouveau une année chaude (on y note la température moyenne mensuelle la plus élevée: $24,5^{\circ} \mathrm{C}$ en juillet). L'année 1989 présente la moyenne annuelle la plus élevée $\left(14,8^{\circ} \mathrm{C}\right)$.

\subsection{Relevés de terrain}

Les mesures physico-chimiques effectuées sont de deux types: d'une part l'analyse ponctuelle et saisonnière de 20 paramètres physico-chimiques (température, conductivité, $\mathrm{pH}$, oxygène dissous, charge organique et minérale) indicateurs de la qualité de l'eau aux 5 stations, d'autre part une surveillance plus particulière de la retenue à l'amont et à l'aval de la centrale portant sur 4 paramètres (température, conductivité, $\mathrm{pH}$, oxygène dissous) mesurés de façon saisonnière sur des transects réalisés en travers du fleuve et à différentes profondeurs.

Fig. 2. - (A) Débits moyens journaliers du Rhône au niveau de la retenue de l'aménagement CNR de Péage-de-Roussillon. (B) Température moyenne journalière de référence du Rhône à l'amont de la centrale et température au canal de rejet enregistrée par les stations de mesures automatiques EDF (voir leur localisation fig. 1C). (C) Ecart thermique, exprimé en valeurs moyennes journalières, entre les deux stations précédentes. (D) Idem (C) mais pour la station Rhône courtcircuité - $(E)$ Puissance moyenne journalière cumulée des deux tranches de la centrale de SaintAlban.

Fig. 2. - (A) Average daily discharge of the Middle Rhône River at Péage-de-Roussillon. (B) Average daily temperature of the Rhône River upstream and immediately downstream from the outflow of heated water of Saint-Alban nuclear plant recorded by EDF automatic measuring stations (see fig. 1C for location). (C) Temperature rise, expressed as average daily values, between the two previous stations. (D) Idem (C) but for the by-passed section. (E) Average daily power of Saint-Alban nuclear plant. 
Les prélèvements de phytoplancton sont effectués de façon saisonnière par traits successifs d'un filet de $60 \mu \mathrm{m}$ de vide de maille en pleine eau dans le chenal afin de remplir un pilulier d'environ $100 \mathrm{ml}$. Ce protocole explique le caractère strictement qualitatif des résultats. Le périphyton est recueilli par grattage d'une surface définie de sédiments (cailloux, galets) ou de débris végétaux (branches mortes) dans un liquide décapant à base de formol et d'acide acétique. L'étude de ces algues ne porte que sur 4 stations, la station 3 (canal de dérivation) étant exclue en raison d'un marnage trop important lié au fonctionnement de l'usine hydroélectrique de Sablons.

L'étude de la végétation aquatique macrophytique a lieu au niveau de la retenue, sur la rive gauche en amont de la centrale et sur les deux rives à l'aval. Les relevés sont réalisés selon la méthode des transects successifs (Balocco-Castella, 1988). Ils sont effectués sur un transect d'une longueur de 1 à $2 \mathrm{~m}$ et sur toute la largeur de l'herbier (5 à $10 \mathrm{~m}$ perpendiculaire à la rive). La liste des espèces présentes est relevée, l'abondance et la sociabilité de chacune d'elles sont estimées par un indice de recouvrement.

Deux techniques de prélévements, complémentaires, sont utilisées afin d'échantillonner au mieux la macrofaune benthique des différents milieux: des substrats artificiels sont déposés près des rives et des draga- ges sont réalisés dans les parties profondes du chenal. Les substrats artificiels utilisés dans cette étude sont composés d'une enveloppe de grillage plastique enfermant des pierres plates et $20 \mathrm{~m}$ de corde sisal. IIs restent immergés pendant quatre semaines, délai moyen nécessaire à leur colonisation optimum par la faune littorale, en place ou dérivante. Selon la nature variable des fonds du fleuve au niveau du secteur d'étude, les dragages sont réalisés à l'aide d'une drague triangulaire ou d'une drague cylindro-conique de type "Railler du Baty ", drague de type océanique réduite à l'échelle $1 / 3$. Ils permettent des prélèvements de la faune du fond proprement dite et du substrat associé. Il est à noter que cette combinaison de techniques et ce type de substrat artificiel sont désormais utilisés dans le cadre du Réseau National de Bassin Rhône-MéditerranéeCorse et du protocole IBGA (IBG adapté aux grands cours d'eau et rivières profondes) (AERMC, 1997).

Enfin, l'ichtyofaune est échantilionnée par pêche électrique selon la méthode des EPA (Nelva et al., 1979; Persat \& Copp, 1990; Persat \& Olivier, 1991) qui, outre la description de la faune piscicole, permet une description multi-ponctuelle de l'habitat par le biais du relevé de diverses caractéristiques du milieu.

Tous ces descripteurs sont échantillonnés de façon saisonnière (4 à 5 passages par an) depuis 1985, voire 1982 pour certains (ARALEPBP, 
1983). La figure 2 donne un résumé du fonctionnement hydrologique et thermique du Rhône, ainsi que du fonctionnement de la centrale, durant la période d'étude.

\subsection{Analyses des données}

Les différents tableaux de données ainsi obtenus ont été traités, pour les descripteurs autres que l'ichtyofaune et la végétation macrophytique, à l'aide d'analyses statistiques inter- et intra-classes (ACP ou AFC) (Dolédec \& Chessel, 1989), qui permettent de prendre en compte, ou au contraire d'éliminer, certains effets liés au processus expérimental.

L'analyse de co-inertie (ACOM) (Chessel \& Hanafi, 1996) s'est avérée la méthode la plus adaptée dans le cas de la végétation aquatique. Elle recherche la co-structure entre plusieurs tableaux et permet de montrer l'existence d'éventuelles ressemblances entre ces tableaux (tableaux de dates ou de stations).

L'analyse factorielle des correspondances décentrée (Dolédec et al., 1995) a été utilisée pour l'analyse des données piscicoles. Cette méthode permet de prendre en considération l'effort d'échantillonnage.

L'ensemble des traitements et des graphiques ont été réalisés avec le logiciel ADE-4 (Thioulouse et al., 1995).

\section{RESULTATS}

\subsection{Physico-chimie}

La charge minérale et organique du Rhône-Moyen à Péage-de-Roussillon présente des variations inter-annuelles mais elle indique toujours un état de pollution chronique. Elle est globalement mise en évidence par les valeurs d'azote ammoniacal, d'azote Kjeldahl et d'orthophosphates, d'une part; par la DBO5 et l'oxydabilité, d'autre part (tabl. I et fig. 3). Si les apports minéraux apparaissent plus importants par rapport à la pollution organique oxydable, ils sont peu à peu assimilés par les grandes dimensions du milieu et l'inertie que cela confère à la masse d'eau vis-à-vis des arrivées extérieures. Même si les concentrations sont souvent supérieures aux seuils classiquement définis (par rapport à la grille de qualité multi-usages de l'Agence de l'Eau Rhône-Méditerranée-Corse, les valeurs moyennes indiquent une pollution modérée pour $\mathrm{NH}_{4}$ et $\mathrm{NK}$ et une pollution importante pour $\mathrm{PO}_{4}$, par exemple), elles sont en réalité représentatives d'un grand cours d'eau de plaine de région tempérée industrialisée.

L'étude des coordonnées de la première valeur propre des différentes analyses statistiques de l'ensemble des résultats du suivi 1982-1994 du site de Saint-Alban montre que l'effet date (saisons et années) est bien supérieur à l'effet station 

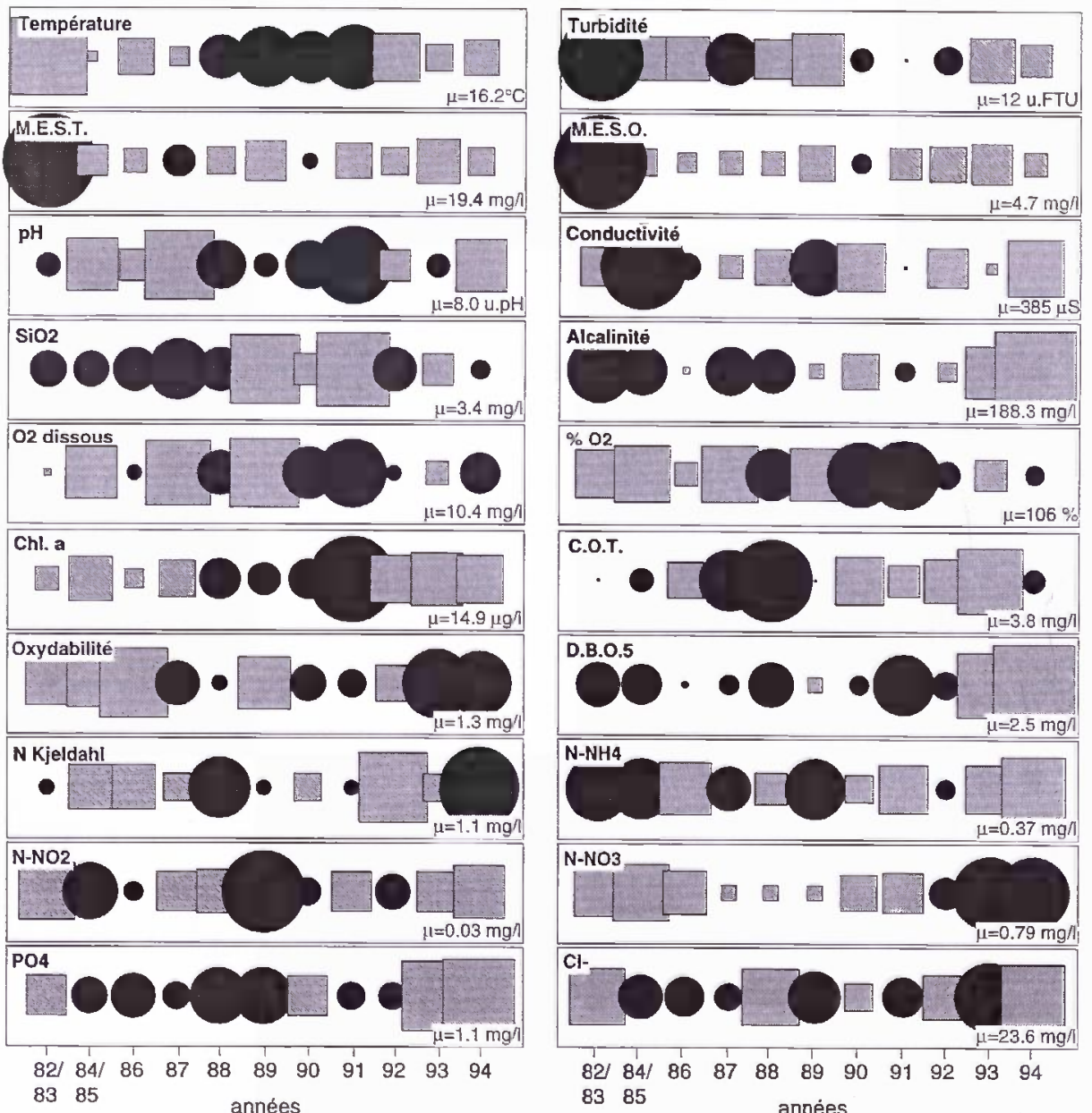

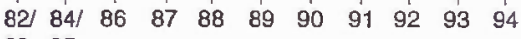

$8385 \quad$ annèes

Fig. 3. - Représentation de l'évolution inter-annuelle 1982-1994 des valeurs normalisées de la moyenne des stations par année des variables physico-chimiques mesurées. Les surfaces des cercles (valeurs supérieures à la moyenne) et des carrés (valeurs inférieures à la moyenne) sont proportionnelles aux données. Les valeurs moyennes générales, toutes stations ef dates confondues, sont indiquées pour chaque paramètre $(\mu)$.

Fig. 3. - Temporal evolution of physico-chemical parameters. Normalized values of the difference to the general mean. The size of circles (values above the mean) and of squares (values below the mean) is proportional to the data. $\mu$ : average values.

(fig. 4A). En effet, si l'on prend comme référence la première valeur propre de l'ACP générale, les analyses intra-, qui mesurent la perte d'in- formation par rapport à la référence, montrent que cette perte est plus importante pour les analyses intra-dates et intra-années que pour celle intra- 


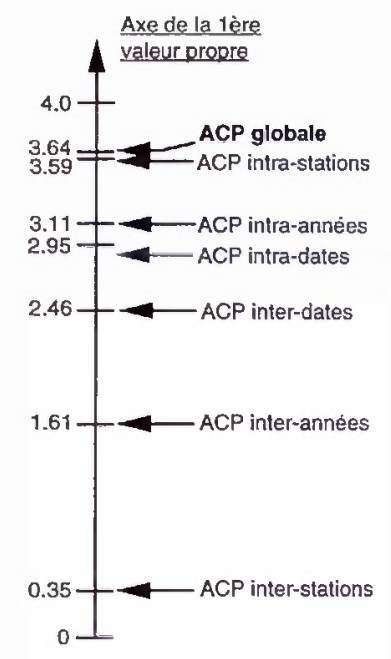

$\mathbf{A}$

\begin{tabular}{|c|c|c|c|}
$\begin{array}{c}\text { Inertie totale de l'ACP } \\
\text { globale }=\mathbf{2 0 . 0}\end{array}$ \\
\cline { 2 - 5 } Facteur & Station & Saison & Année \\
\hline ACP inter-classes & 0.54 & 3.23 & 4.34 \\
\hline ACP intra-classes & 19.46 & 16.77 & 15.66 \\
\hline
\end{tabular}
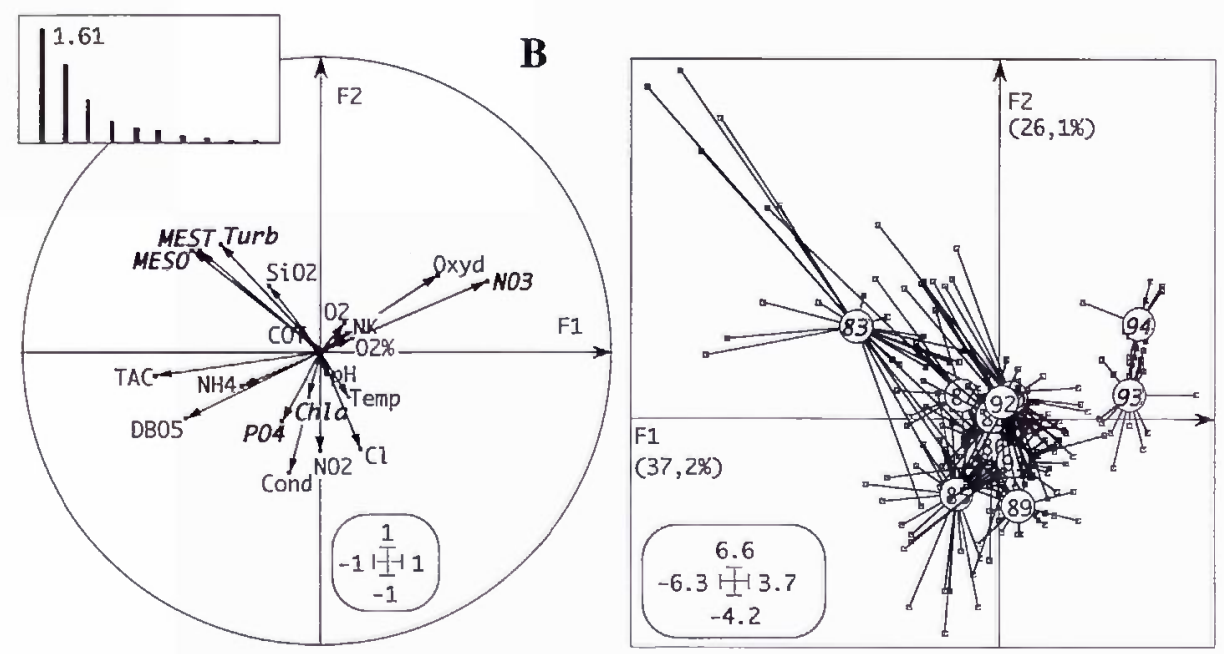

Fig. 4. - Etude des paramètres physico-chimiques. (A) Parts d'influence des facteurs spatiaux et temporels résultant des ACP inter- et intra-classes exprimées par le pourcentage d'inertie et les coordonnées de la première valeur propre des différentes analyses. (B) ACP inter-années : graphe des valeurs propres, cercle de corrélation des variables et carte factorielle des relevés.

Fig. 4. - Data analysis on physico-chemical parameters. (A) Influence of spatial and temporal factors according to the between and within Principal Components Analyses : percentage of inertia and coordinates of the first eigenvalue of each analysis. (B) Between-years Principal Components Analysis.

stations; tandis que les analyses inter-, qui mesurent le gain d'information, montrent un effet saison supérieur à l'effet station. Ceci signifie que des événements tels que l'hydrologie (qui influe corrélativement sur le volume 
des apports) ou l'arrêt de certains rejets industriels à l'amont du site sont des événements majeurs dans la dynamique physico-chimique du secteur de Péage-de-Roussillon. Les variables tour à tour prépondérantes sont la charge minérale et organique et la charge en suspension en "période froide", le facteur thermique et les paramètres liés à la production et à la consommation d'oxygène dissous en "période chaude".

L'ACP inter-années différencie deux périodes (fig. 4B) :

(1) la période 1982-1992 durant laquelle les teneurs en phosphates, en ammoniaque, en chlorophylle a et la DBO5 sont souvent supérieures à la moyenne de la chronique d'étude, tandis que celles en nitrates sont inférieures;

(2) les années 1993-1994, qui se caractérisent par une nette diminution des teneurs en phosphates probablement liée à l'arrêt de divers rejets industriels en amont du site à la fin de l'année 1992, une baisse des teneurs en chlorophylle a planctonique et une augmentation des teneurs en nitrates.

On note de ce fait un fort accroissement corrélatif du rapport nitrates/phosphates, qui passe de 3 en moyenne sur la période 1982-1992 à 20-30 en 1993 et 1994. Sur l'ensemble de la chronique 1985-1994, les nitrates ne limitent pas la croissance algale planctonique; une faible corrélation inverse $(r=-0,365)$ est en effet notée entre les concentrations en nitrates et en chlorophylle a de la re- tenue aux stations 1 et 2. La croissance des algues pourrait être en revanche limitée par les phosphates, ce qui expliquerait la baisse de chlorophylle a. Toutefois, Crouzet \& Amezal (1982) considèrent que des teneurs de 0,1 à $0,15 \mathrm{mg} / \mathrm{l}$ de phosphates (les valeurs 93-94 étant voisines, voire supérieures à ce seuil) permettent d'atteindre un taux de $30 \mu \mathrm{g} / \mathrm{l}$ de chlorophylle a.

La période $82-83$, représentée par l'année 83 sur la fig. $4 \mathrm{~B}$, se caractérise par une hydrologie excédentaire (hydraulicité $=1,3$, fig. 1D) qui explique les fortes teneurs en matières en suspension totales et organiques et les valeurs de turbidité très élevées. Les variations de la qualité physicochimique générale sont donc soit dues à l'hydrologie, soit en liaison avec un changement dans différents apports.

Des transects physico-chimiques pratiqués en largeur et en profondeur, à l'amont et à l'aval de la centrale, dans la retenue de St-Pierre-de-Bœuf montrent que la retenue peut présenter une stratification naturelle lors d'épisodes climatiques particuliers, tel qu'au printemps 1991. D'autre part, selon les conditions hydrologiques et éoliennes, la lame d'eau réchauffée issue de la centrale diffuse plus ou moins largement et profondément sur le plan d'eau de la retenue (station 2) et n'est pas totalement évacuée par le canal de dérivation. Une partie, plus ou moins diluée, est en effet présente en permanence devant le barrage de retenue et le Rhône court-circuité (station 2'), le réchauf- 
fement étant sensible aussi bien en largeur qu'en profondeur, malgré le début de mélange des masses d'eau (fig. 5 et tabl. II). Toutefois, à l'exception de la température, les autres mesures montrent globalement une grande homogénéité des valeurs entre les stations et les profondeurs pour une même date. La teneur en oxygène reste notamment satisfaisante, en particulier lors des périodes estivales et/ou d'étiage marqué: à l'exception de deux dates, où les valeurs étaient comprises entre 6 et $7 \mathrm{mg} / \mathrm{l}$ selon la profondeur, les teneurs mesurées lors des différentes périodes estivales sont toujours $\geq 9 \mathrm{mg} / \mathrm{l}$, voire $\geq 10 \mathrm{mg} / \mathrm{l}$, quelle que soit la station ou la profondeur.

Dans le cadre du programme de surveillance hydroécologique du site de Saint-Alban, EDF mesure en continu divers paramètres physico-chimiques en plusieurs points de l'aménagement hydroélectrique (voir "sta- tions EDF" fig. 1C). L'exploitation des chroniques de température d'eau relevées en amont et au canal de rejet de la centrale (fig. 2C) montre un écart thermique moyen de près de $6{ }^{\circ} \mathrm{C}$ sur la période 1988-1994 (tabl. III). Par ailleurs, la température moyenne mensuelle dans ce canal ne descend jamais en dessous de $11^{\circ} \mathrm{C}$.

Les enregistrements pratiqués au niveau de la partie court-circuitée (fig. 2D) font apparaitre un écart moyen journalier et un écart moyen annuel voisins $\left(1,6^{\circ} \mathrm{C}\right)$ par rapport au Rhône à l'amont de la centrale (tabl. III). Toutefois, les parts respectives du réchauffement naturel de ce milieu et de l'influence thermique du rejet de la centrale sont difficilement appréciables. Selon un calcul effectué par EDF DER, pour une gamme de débit de 10 à $20 \mathrm{~m}^{3} / \mathrm{s}$, l'échauffement maximum lié aux échanges thermiques avec l'atmosphère peut atteindre 5 à $6{ }^{\circ} \mathrm{C}$ en période estivale. L'influence

Tableau II. Résumé des écarts moyens de température (valeurs mini. et maxi. entre parenthèses), par rapport à la station amont (pk 47), mesurées en surface à l'aval du site de Saint-Alban lors des campagnes de transects physicochimiques.

Table II. Summary of average differences of temperature (minimal and maximal values in brackets), compared to upstream data (kilometric point 47), measured at the surface downstream from Saint-Alban power plant during the physico-chemical sampling plots.

\begin{tabular}{lcc|}
\hline Station hydroécologique : & Station 2 & Station $\mathbf{2}^{3}$ \\
Localisation : & $\begin{array}{c}\text { Retenue } \\
\text { pk } 49\end{array}$ & $\begin{array}{c}\text { Retenue } \\
\text { pk } 50,5\end{array}$ \\
Hiver & $2,0(0-5,1)$ & $1,4(0,7-1,9)$ \\
Printemps & $3,5(1,5-5,7)$ & $3,2(2,5-4,1)$ \\
Eté & $2,2(1,1-3,1)$ & $1,7(1,3-2,0)$ \\
Automne & $1,9(0-4,1)$ & $1,7(1,0-2,3)$ \\
Ecart moyen général & $\mathbf{2 , 4}(0,7-4,3)$ & $\mathbf{2 , 0}(1,5-2,5)$ \\
\hline
\end{tabular}


- Station 2 - pk 49 -

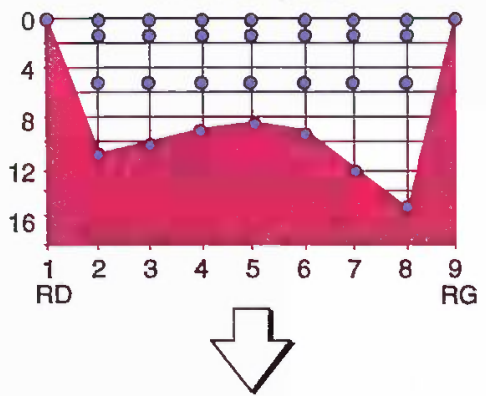

- Station 2 .

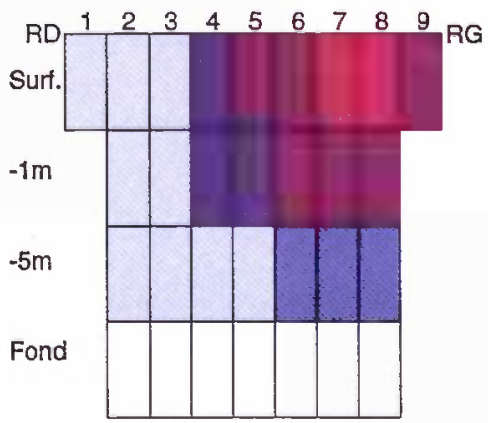

Rejet St-Alban

- Station 2' - pk 50,5 -

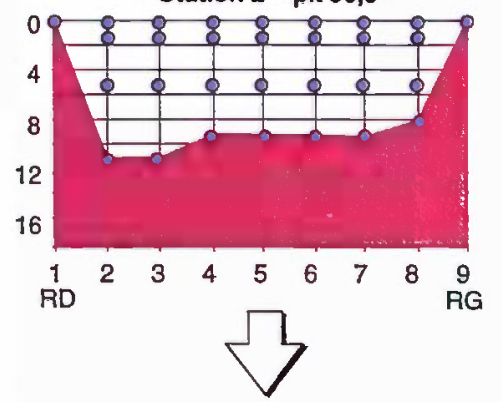

- Station 2' -

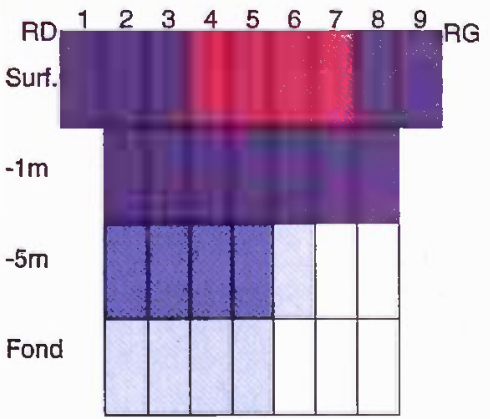

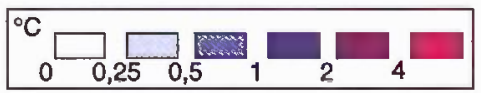

Fig. 5. - Ecarts moyens de température enregistrés aux stations 2 et 2' situées à l'aval de la centrale (voir localisation fig. 1C) par rapport à la température de référence amont, lorsque la centrale fonctionnait (27 campagnes sur la période 1986-1994). Valeurs globales toutes saisons confondues. Les points de mesure sont localisés par la profondeur et la distance à la rive droite exprimés en mètres.

Fig. 5. - Average differences of temperature between stations 2 and 2' downstream from the plant (see fig. $1 \mathrm{C}$ for location) compared to the upstream temperature, when the plant operated (27 campaigns over the period 1986-1994), all seasons pooled.

de la centrale est ainsi soit non significative, soit limitée $\left(<1^{\circ} \mathrm{C}\right)$, soit à rechercher dans les valeurs extrêmes, en particulier celles enregistrées en période automnale $\left(4,2^{\circ} \mathrm{C}\right.$ en novembre 94, par exemple). Lorsqu'elle est sensible, elle agit principalement par un relèvement des minima. 
Tableau III. Valeurs caractéristiques de la température du Rhône enregistrèe aux différentes stations de mesures automatiques EDF du site de Saint-Alban (cf Fig. 1C pour leur localisation). Valeurs moyennes journalières 1988-1994.

Table III. Significant temperatures of the Rhône River recorded by EDF automatic measuring stations of Saint-Alban power plant (see Fig. 1C for location). Average daily data 1988-1994.

\begin{tabular}{|c|c|c|c|c|}
\hline Station EDF : A & Canal rejet & Aval & Rhône court-circuité & \\
\hline Localisation : & $\begin{array}{l}\text { Retenue } \\
\text { pk } 47,5\end{array}$ & $\begin{array}{l}\text { Retenue } \\
\text { pk } 48,5\end{array}$ & $\begin{array}{l}\text { Canal } \\
\text { pk } 55\end{array}$ & $\begin{array}{l}\text { R.C.C. } \\
\text { pk } 54\end{array}$ \\
\hline Temp. moyenne & 13,9 & 19,7 & 14,7 & 15,5 \\
\hline Temp. mini. & 2,2 & 5,1 & 3,5 & 4,3 \\
\hline Temp. maxi. & 25,8 & 32,0 & 27,8 & 32,5 \\
\hline $\begin{array}{l}\text { Ecart } \\
\text { moyen/amont }\end{array}$ & I & 5,8 & 1,0 & 1,6 \\
\hline Ecart mini. & & & non significatif* & \\
\hline Ecart maxi. & $\begin{array}{c}/ \\
(22 / 11 / 88)\end{array}$ & $\begin{array}{c}11,9 \\
(15 / 03 / 93)\end{array}$ & $\begin{array}{c}7,8 \\
(22 / 08 / 92)\end{array}$ & 11,5 \\
\hline
\end{tabular}

* non significatif, car valeurs négatives pour lesquelles il est difficile de faire la part avec les possibles problèmes dus aux sondes.

Ainsi, en dehors de l'échauffement, particulièrement net à l'aval immédiat du site (station aval du programme de surveillance hydroécologique), le rejet de Saint-Alban ne semble pas perturber outre mesure les divers processus chimiques et la composition physico-chimique des stations amont et aval centrale (stations 1 et 2) est très voisine.

\subsection{Les algues du phytoplancton et du périphyton}

La dynamique des peuplements phytoplanctoniques et périphytiques d'un grand cours d'eau est difficile à appréhender: en ce qui concerne le phytoplancton, peu d'études de ce type étaient référencées dans la bibliographie jusqu'au milieu des années 80 (Descy, 1992), tandis que les études portant sur le périphyton s'intéressent essentiellement à la biomasse, à l'exception du travail de Lair et al. (1996) sur la Loire. La variabilité non expliquée par les analyses est importante. Cela est dû essentiellement au caractère accidentel de beaucoup de taxons.

Les Diatomées et les Chlorophycées (dans des proportions voisines) dominent largement le peuplement phytoplanctonique global et représen- 
tent près de $80 \%$ des 291 taxons répertoriés, tandis que les Cyanophycées représentent près de $10 \%$ des taxons (fig. 6A). La plupart de ces taxons sont peu fréquents voire rares: seulement 13 espèces (11 Diatomées, 1 Chrysophycée et 1 Chlorophycée) sont constantes sur l'ensemble de la période d'étude, c'est-à-dire présentes dans plus de $50 \%$ des relevés (dont Aulacoseira granulata et Synedra acus dans plus de $95 \%$ de ces relevés), 19 autres espèces sont accessoires (présence dans 25 à $50 \%$ des relevés), tandis que les 259 autres taxons sont sporadiques (présence dans moins de $25 \%$ des relevés). La représentation de ces relevés sur le plan F1×F2 de l'AFC inter-années (fig. 6B) montre une opposition sur le premier axe entre les années 1987-88 et les années 1990-94; 1989 étant en position intermédiaire. Le deuxième axe permet de distinguer 1993 et 1994 des autres années. L'hydraulicité faible du Rhône en 1989 (coefficient de 0,7) et l'augmentation des températures qui en découle pour le premier cas, la réduction des apports phosphatés à partir de 1992 pour le second cas, sont sans doute les facteurs explicatifs de la modification du peuplement phytoplanctonique. D'un point de vue trophique, la majorité des taxons de ces différentes périodes est caractéristique d'eaux eutrophes au sens large (méso-eutrophes à eutrophes), composée d'espèces ubiquistes et opportunistes comme c'est souvent le cas dans les cours d'eau de plaine à ten- dance eutrophe (Descy, 1992 et 1993; Del Giorgio et al., 1991).

Le peuplement périphytique du secteur d'étude est constitué de 247 taxons dont une large majorité de Diatomées (205 taxons). Les Chlorophycées (23 taxons), les Desmidiées (10 taxons) et les Cyanophycées (7 taxons) viennent ensuite (fig. 7A). Comme pour le phytoplancton, la plupart des taxons sont peu fréquents voire rares: seulement 16 espèces (toutes des Diatomées) sont constantes sur l'ensemble de l'étude, 17 sont accessoires, tandis que les 214 autres sont sporadiques. 5 espèces (Nitzchia dissipata, Navicula trivialis, Melosira varians, Navicula sp. et Cocconeis pediculus) sont présentes dans plus de $80 \%$ des relevés. Le plan $\mathrm{F} 1 \times \mathrm{F} 2$ de l'analyse "inter-années " montre sur le premier axe une opposition assez nette entre 1987 et 1988 d'une part et les autres années d'autre part (fig. 7B). La fin des années 80 constitue de nouveau une charnière dans l'évolution du peuplement périphytique. Les années 1989 à 1994 se répartissent selon l'axe F2 mettant en évidence une lente évolution du peuplement durant cette période. Les peuplements observés en 1987 présentent la particularité de n'être composés que de Diatomées, les Cyanophycées et les Chlorophycées n'apparaissant qu'en 1988 et surtout en 1989.

Les peuplements phytoplanctoniques se renouvellent constamment en raison de la nature fluviale du site d'étude, ce qui tend à déstructurer leur organisation spatiale et tempo- 

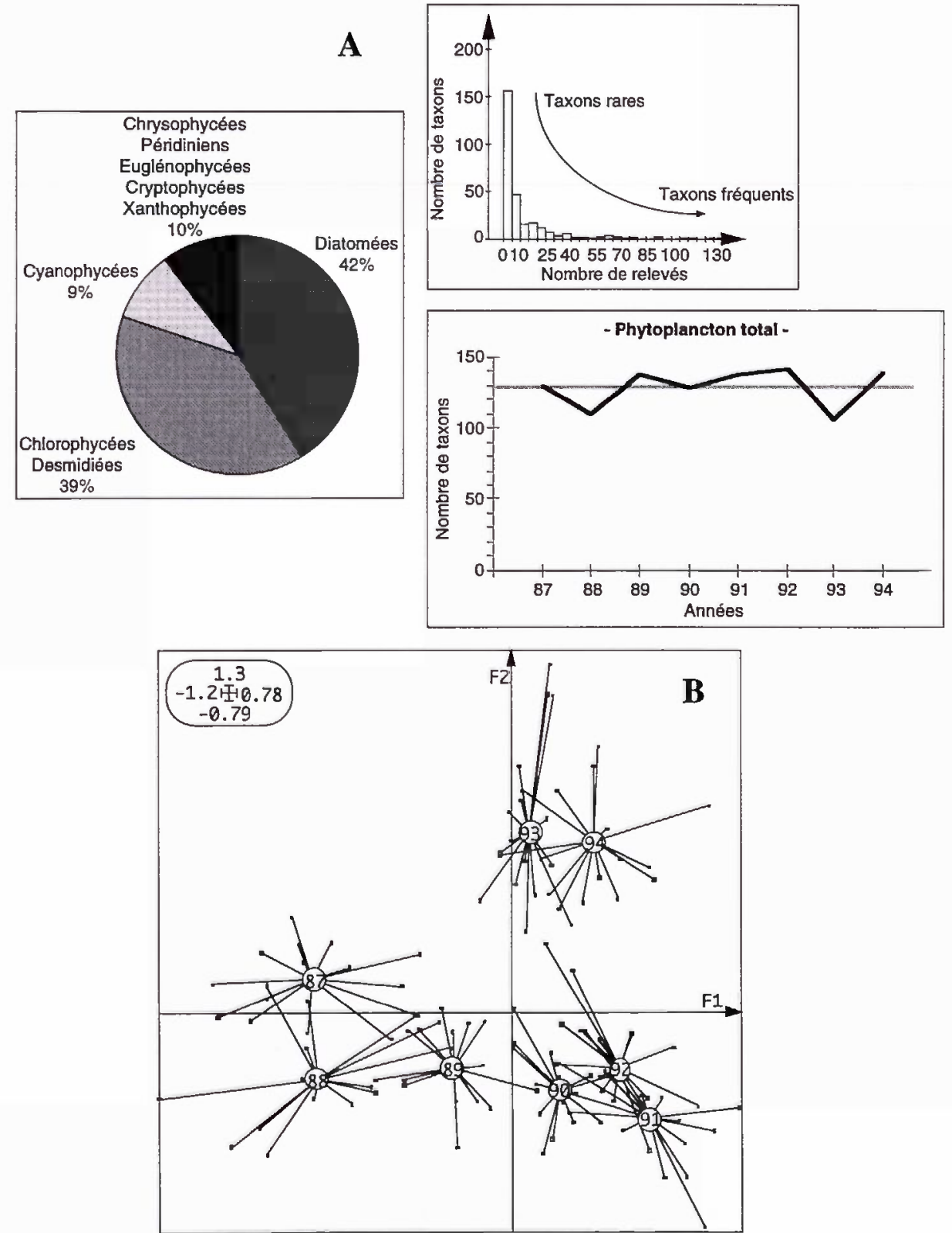

Fig. 6. - Etude des algues phytoplanctoniques. (A) Composition du peuplement, histogramme de fréquence des taxons inventoriés et évolution inter-annuelle de la richesse taxonomique totale (le trait horizontal indique la valeur moyenne de la période 87-94). (B) Carte factorielle des relevés de l'AFC inter-années.

Fig. 6. - Structure and evolution of phytoplanktonic communities. (A) Abundance, frequency and yearly richness of the communities (the horizontal line indicates the average value 1987-1994). (B) Between-years Correspondance Analysis. 


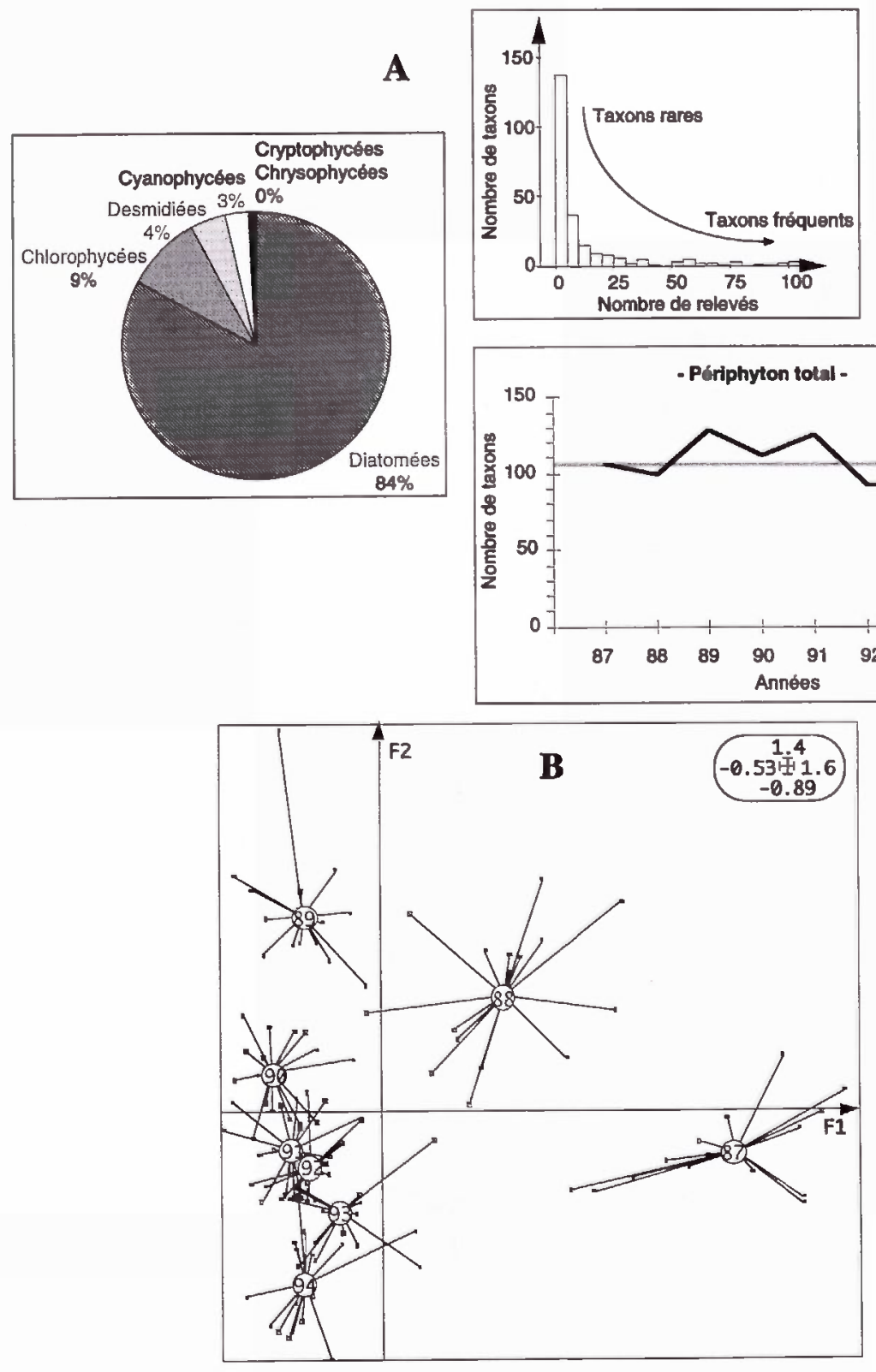

Fig. 7. - Etude des algues périphytiques. (A) Composition du peuplement, histogramme de fréquence des taxons inventoriés et évolution inter-annuelle de la richesse taxonomique totale (le trait horizontal indique la valeur moyenne de la période 87-94). (B) Carte factorielle des relevés de l'AFC inter-années.

Fig. 7. - Structure and evolution of periphytic communities. (A) Abundance, frequency and yearly richness of the communities (the horizontal line indicates the average value 1987-1994). (B) Between-years Correspondance Analysis. 
relle. Inversement, le périphyton apparait plus caractéristique de la station où il est prélevé.

L'hydrologie, de par les conditions générales du site d'étude comme par celles particulières à certaines stations (stations 4 et 5 notamment), les caractères chimiques de l'eau liés aux apports du bassin (apports proches ou éloignés) ansi que les caractères physiques des eaux (température, MES...) sont des éléments très structurants pour le peuplement global du secteur. Cela se vérifie au niveau du rôle clé joué par l'année 1989 (faible hydraulicité et plus forte température), d'une part, par la baisse de la richesse à partir de 1992 (surtout sensible au niveau du périphyton) sans doute liée à une modification des apports nutritifs (notamment en éléments phosphorés), d'autre part. De manière identique, Lair \& Sargos (1993) ont montré sur la Loire le rôle du débit et de la température en absence de limitation des nutriments dans le contrôle de la biomasse algale, mais aussi l'importance de la qualité de l'eau dans certains cas.

\subsection{La végétation macrophytique}

Si le secteur d'étude semble globalement homogène dans le temps et dans l'espace, certaines tendances peuvent toutefois être dégagées :

- La communauté des macrophytes aquatiques est dominée par six espèces abondantes (Potamogeton pectinatus, Lemna minor, Spirodela polyrhiza, Ceratophyllum demersum, Myriphyllum demersum, Potamogeton nodosus) dont les modes de distribution génèrent la plus grande part de la variabilité.

- Le groupement à Potamogeton pectinatus, associé à Lemna minor et Spirodela polyrhiza est le groupement dominant sur toute la durée d'étude. Espèce d'eau plutôt alcaline et assez résistante à la pollution, caractéristique d'eau eutrophe, Potamogeton pectinatus est nettement l'espèce la plus abondante et la plus fréquente sur l'ensemble du secteur d'étude.

- II apparait secondairement des différences entre stations principalement liées à la dominance des espèces fixées (les potamots et les myriophylles), des espèces situées entre deux eaux (Ceratophyllum demersum) ou des espèces totalement flottantes (Spirodela polyrhiza, Lemna minor). Ces différences sont fonction de l'hydrologie annuelle et de l'extension ou non des algues filamenteuses, extension en particulier régie par les crues.

La représentation des corrélations relatives à chaque sous-tableau (chaque année) met en évidence la typologie des années (fig. 8A). La représentation des corrélations sur le plan F1×F2 permet d'affirmer que la co-structure des tableaux-années est forte, c'est-à-dire que la distribution spatiale des espèces dans le secteur d'étude (les trois stations confondues) présente les mêmes caractéristiques 
$\mathbf{A}$

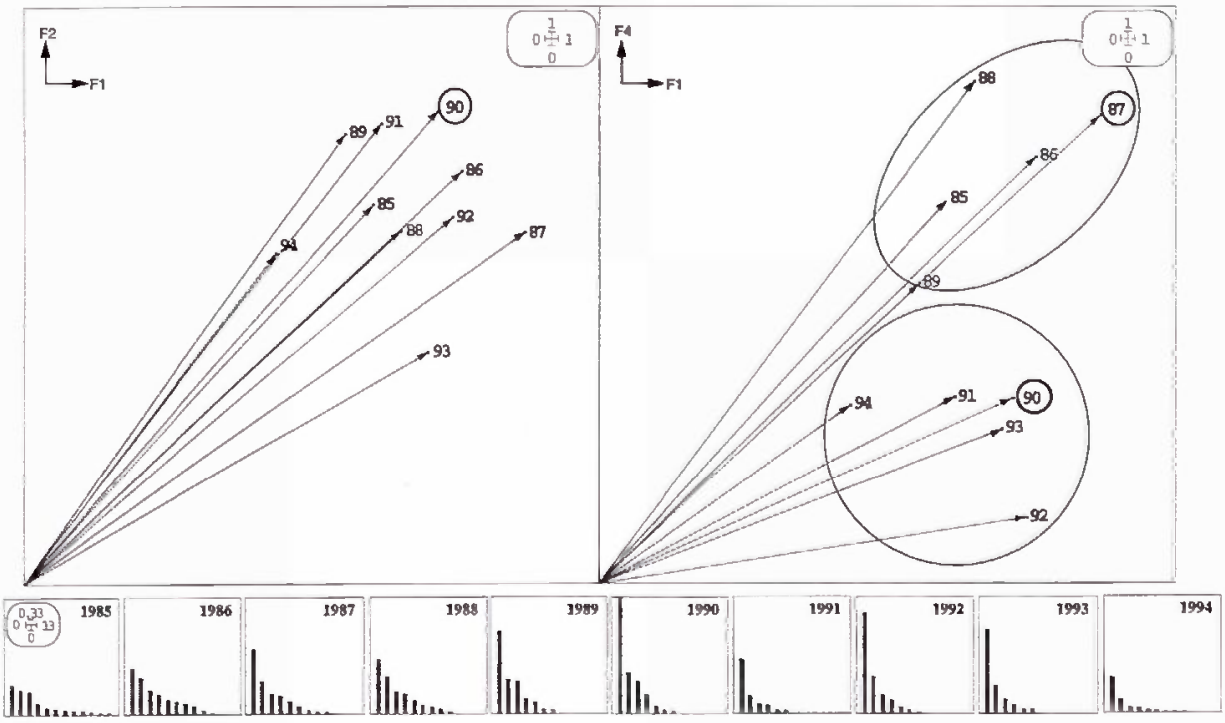

B

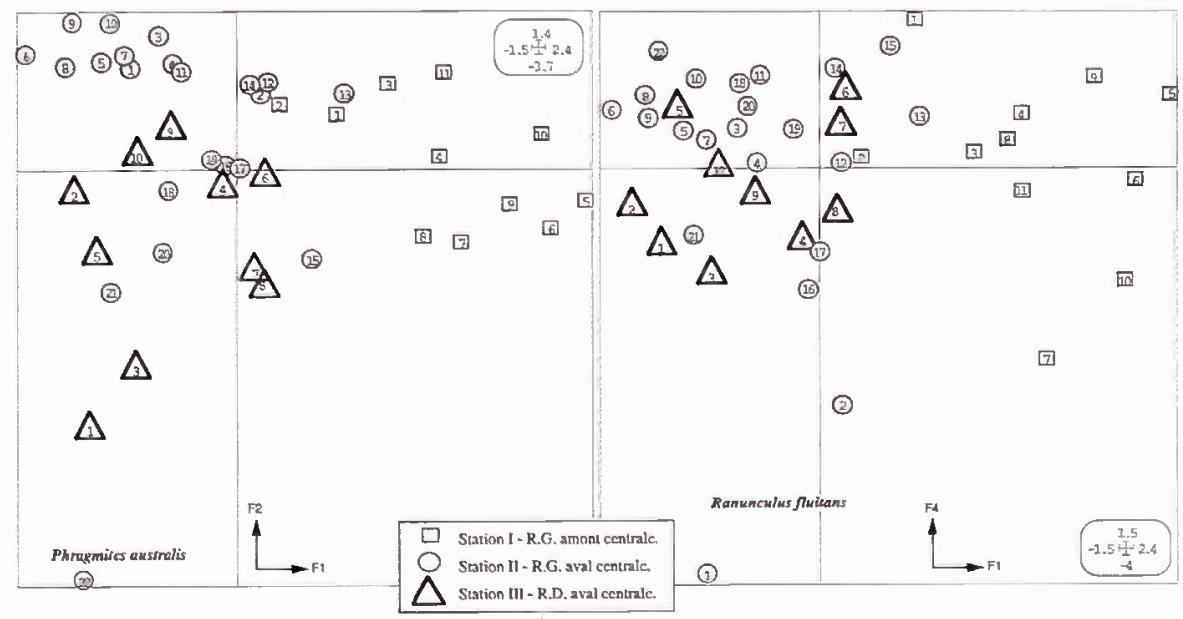

Fig. 8. - Etude de la végétation macrophytique. (A) Evolution temporelle : représentation des corrélations issues de l'ACOM des tableaux annuels transects $\times$ espèces et graphes des valeurs propres de chaque analyse. (B) Evolution spatiale: cartes factorielles des relevés (transects) de l'ACOM transects $\times$ espèces.

Fig. 8. - Structure and evolution of the aquatic macrophytes. (A) Temporal evolution : correlations resulting from the analysis of yearly data tables "sampling plots $\times$ species" and eigenvalues graph of each analysis. (B) Spatial evolution: factorial maps of the "sampling plots $x$ species" analysis. 
au cours des dix années. L'absence de variabilité temporelle de l'organisation spatiale du site ne signifie toutefois pas qu'il ne se passe rien et que les abondances des espèces sont toujours les mêmes, mais les variations inter-annuelles, si elles existent, sont les mêmes dans les trois stations, à quelques détails près. Sur le plan $\mathrm{F} 1 \times \mathrm{F} 4$, c'est à dire pour un niveau de variabilité moindre, il apparaît deux groupes d'années: les années 85 à 89 d'une part, et les années 90 à 94 d'autre part. Ces résultats montrent que 1989 est une année "charnière", à partir de laquelle la structure commence à changer.

Du point de vue spatial, la distinction de la rive gauche à l'aval de la centrale dans les années 80 est réelle mais ne constitue pas un phénomène particulièrement significatif : la part de la variabilité est faible et ne concerne que deux espèces, respectivement caractérisées par les axes 2 et 4 de l'analyse (fig. 8B). Ce sont d'une part Phragmites australis, dont quelques individus isolés sont rencontrés immédiatement à l'aval du site à partir de 1989 (transect de relevés $n^{\circ} 22$ avec abondance de dépôts limoneux), et d'autre part Ranunculus fluitans, dont la présence était significative entre 1985 et 1988 à l'entrée du canal de dérivation (transects de relevés $n^{\circ} 1$ et 2 avec une vitesse du courant sensible).

\subsection{Les macroinvertébrés benthiques}

Au total 143 taxons appartenant à 82 familles ont été récoltés les deux techniques de prélèvement confondues, respectivement 127 pour les substrats artificiels seuls et 101 pour les dragages. Sur ce total général de 143 taxons, 46 sont "accidentels" (représentés que par 1 ou 2 individus seulement ou présents dans 1 ou 2 prélévements), 44 sont exclusifs des substrats (ce sont essentiellement des larves d'Insectes) et 17 sont exclusifs des dragages. Les deux ensembles fonctionnels les plus courants, le canal de fuite et le Rhône court-circuité, ont les plus fortes richesses taxonomiques, avec une relative homogénéité de distribution entre les substrats et les dragages. Les richesses toujours plus élevées des substrats artificiels, par rapport aux dragages, mettent en évidence l'importance biologique des zones rivulaires (Fruget, 1994), mais aussi le caractère inhospitalier du chenal de certains milieux dû à une sédimentation excessive (retenue et plan d'eau).

D'un point de vue temporel, une décroissance de la richesse taxonomique est enregistrée à la fin des années 80 et au début des années 90 , puis un recouvrement partiel à partir de 1992 (voir fig. 10). Cette évolution est sous la dépendance de facteurs 
hydroclimatiques (succession des périodes d'étiage et de crue, influant corrélativement sur la température de l'eau). Ceci est tout à fait en accord avec le concept de perturbation-diversité et la théorie des perturbations moyennes (Connell, 1978; Ward \& Stanford, 1983) : en conditions stables (années à faible hydrologie) la richesse spécifique est basse (années 1989-1991), elle s'accroit avec l'existence de fluctuations hydrologiques (années 1993-94).

Les Mollusques constituent le groupe faunistique le plus diversifié, suivi des Trichoptères, des Odonates et des Diptères. Par contre, en considérant ces mêmes groupes faunistiques selon l'abondance relative, la macrofaune benthique du Rhône à Péage-de-Roussillon est principalement dominée par les Oligochètes (quelque soit la technique de prélévement) et, de façon secondaire, par les Diptères Chironomidés et les Aselles. Les taxons les plus significatifs sont principalement les Oligochètes, les Diptères Chironomidés et les Aselles, puis, dans un second temps, des Mollusques, des Achètes et des Turbellariés. L'ordre d'Insectes le plus significatif est celui des Trichoptères, parmi lesquels Ecnomus tenellus représente près de $3 / 4$ des individus. Toutefois, si elles ne sont pas significativement abondantes, certaines larves d'Insectes (autres que les Diptères) sont régulièrement présentes dans les relevés (Trichoptère Ecnomus tenellus, Odonates Ischnura elegans, Platycnemis pennipes et Cercion lindeni, en particulier).
L'analyse statistique des résultats fait apparaitre des différences spatiales plus nettes que les différences d'ordre temporel, saisonnier ou interannuel (fig. 9A). L'importance de l'hétérogénéité de l'habitat (type de prélévement: substrat artificiel ou dragage, rive ou chenal, limon ou galets) est fondamentale dans le cas de la faune (fig. 9B). L'étude spécifique de l'analyse inter-annuelle (fig. 9C) permet de caractériser l'évolution constatée au cours du suivi à longterme : il en ressort une nette partition entre les années antérieures à 1989 et les années postérieures à 1989. L'évolution au cours de la chronique de paramètres descriptifs des conditions environnementales (débit et température moyennes annuelles) permet d'expliquer cela: ainsi, les conditions de l'année 1989 (plus faible hydrologie et forte température) a dynamisé l'installation et l'expansion de certaines espèces tel que le Trichoptère Ecnomus tenellus (fig. 10), taxon potamo-lénitique. Ce Trichoptère est en effet une espèce de cours d'eau calmes, tels que la Saône ou la Meuse (Tachet et al., 1988; Meurisse-Genin et al., 1987), qui a tiré profit des faibles débits des années 1989-1991 pour augmenter ses populations sur le Rhône aménagé et $s^{\prime} y$ maintenir depuis.

L'écrasante majorité de taxons a une affinité pour les vitesses de courant réduites (individus limno-rhéophiles), attestant ainsi de la profonde transformation mésologique du fleuve par l'aménagement CNR, qui a favorisé les espèces potamo-lénitiques. 


\begin{tabular}{|c|c|c|c|c|c|c|}
\hline \multirow{2}{*}{ Axe de la 1ère } & & \multicolumn{3}{|c|}{$\begin{array}{l}\text { Inertie totale de l'AFC } \\
\text { globale }=3.13\end{array}$} & \multirow[b]{2}{*}{ Techniq } & \multirow[b]{2}{*}{ Sta.Tec } \\
\hline & Analyse Facteur & Station & Saison & Année & & \\
\hline valeur propre & AFC inter-classes & 0.28 & 0.14 & 0.22 & 0.11 & 0.50 \\
\hline 0.30 & AFC intra-classes & 2.85 & 2.99 & 2.63 & 3.02 & 2.63 \\
\hline
\end{tabular}
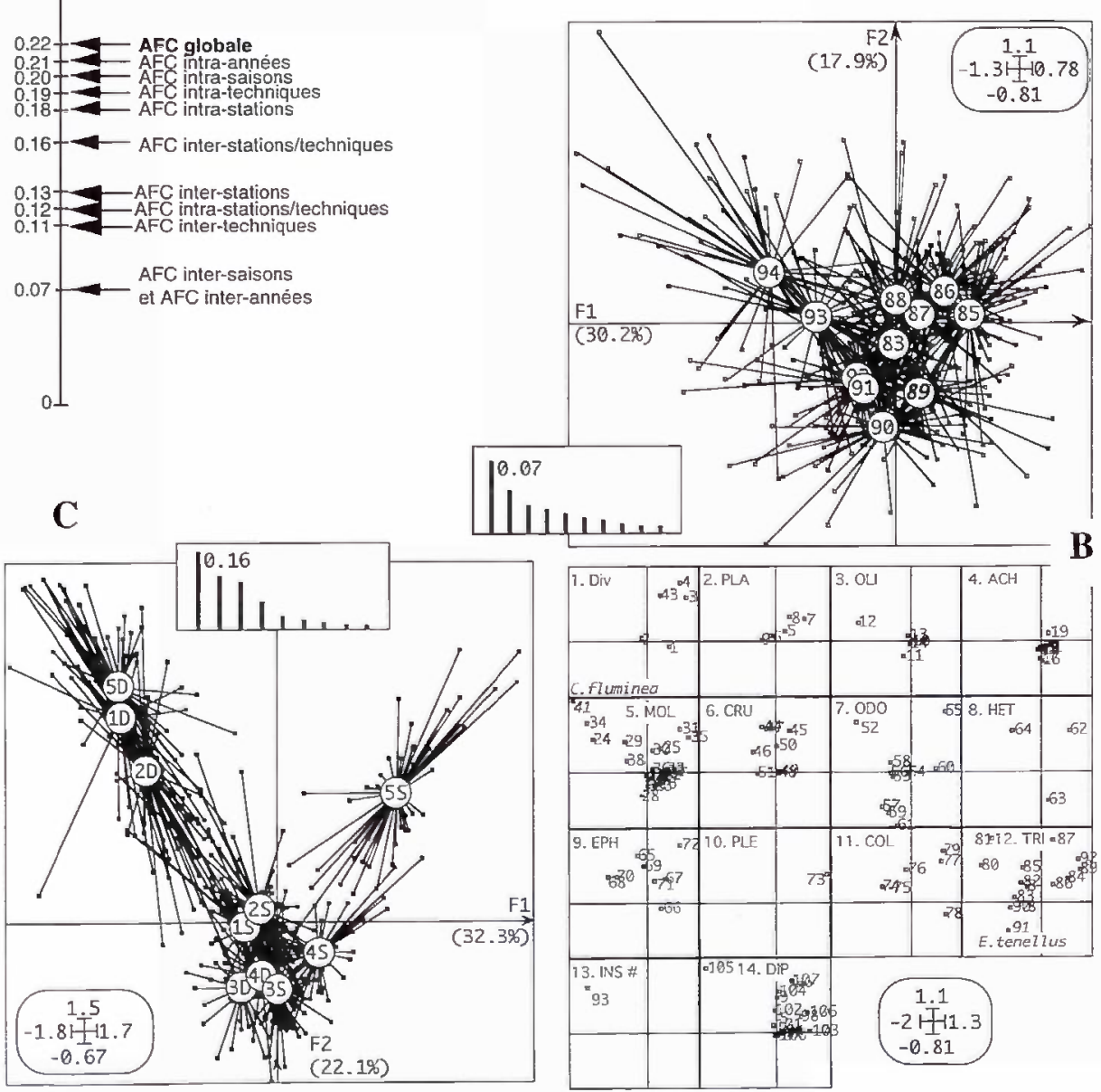

Fig. 9. - Etude de la macrofaune benthique. (A) Parts d'influence des facteurs spatiaux et temporels résultant des AFC inter-et intra-classes exprimées par le pourcentage d'inertie et les coordonnées de la première valeur propre des différentes analyses. (B) AFC inter-années: graphe des valeurs propres, cartes factorielles des relevés et des taxons distingués par groupes faunistiques. (C) AFC inter-stations/techniques: graphe des valeurs propres et carte factorielle des relevés $\left(x: n^{\circ}\right.$ de la station; $S$ : substrat artificiel; $D$ : dragage).

Fig. 9. - Evolution of benthic communities. (A) Influence of spatial and temporal factors according to the between and within Principal Components Analyses : percentage of inertia and coordinates of the first eigenvalue of each analysis. (B) Between-years Correspondance Analysis. (C) Between-stations/techniques Correspondance Analysis ( $x$ : station number; $S$ : artificial substrate; D : dredge). 
Ce constat n'est pas propre au seul aménagement de Péage-de-Roussillon, mais à l'ensemble du BasRhône (Fruget, 1991). Outre les conditions locales, des phénomènes temporels expliquent également la dynamique des différentes populations, en particulier les variations climatiques inter-annuelles. La figure 11 montre les changements d'abondance enregistrées par différentes espèces sur l'ensemble de la période considérée. Quatre types d'évolution biologique peuvent ainsi être mis en parallèle avec les paramètres hydrologie et température. Deux se font dans le sens d'une simple croissance ou décroissance tandis que les deux autres sont suivis d'un retour à l'abondance initiale, l'année 1989 servant généralement de date charnière. Dans ces conditions, les crues sont le facteur de contrôle principal de la structure des peuplements benthiques. Le phénomène de redistribution et d'extension de certains organismes

Fig. 10. - Relation entre des paramètres descripteurs des macroinvertébrés (coordonnées F1 inter-années, richesse taxonomique totale annuelle et abondance moyenne par prélévement d'Ecnomus tenellus) et deux paramètres environnementaux (débit moyen annuel et température moyenne annuelle du Rhône à l'amont de la centrale).

Fig. 10. - Structure of benthic communities. Relationship between some biological factors ( $F 1$ between-years coordinates, yearly total taxonomic richness, average abundance of Ecnomus tenellus) and two environmental parameters (yearly discharge and temperature of the Rhône River upstream from the power plant).
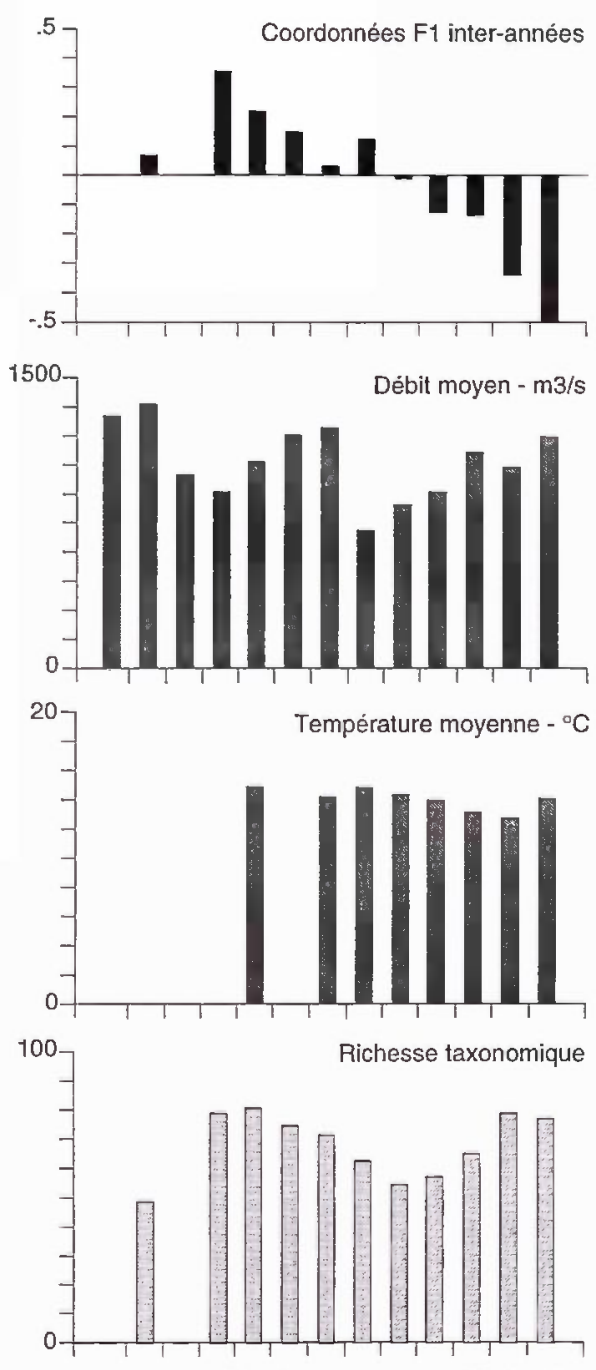

707 Abondance moyenne $\mathrm{d}^{\top} E$. tenellus

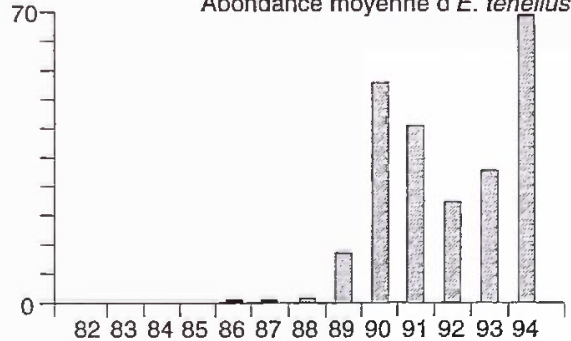



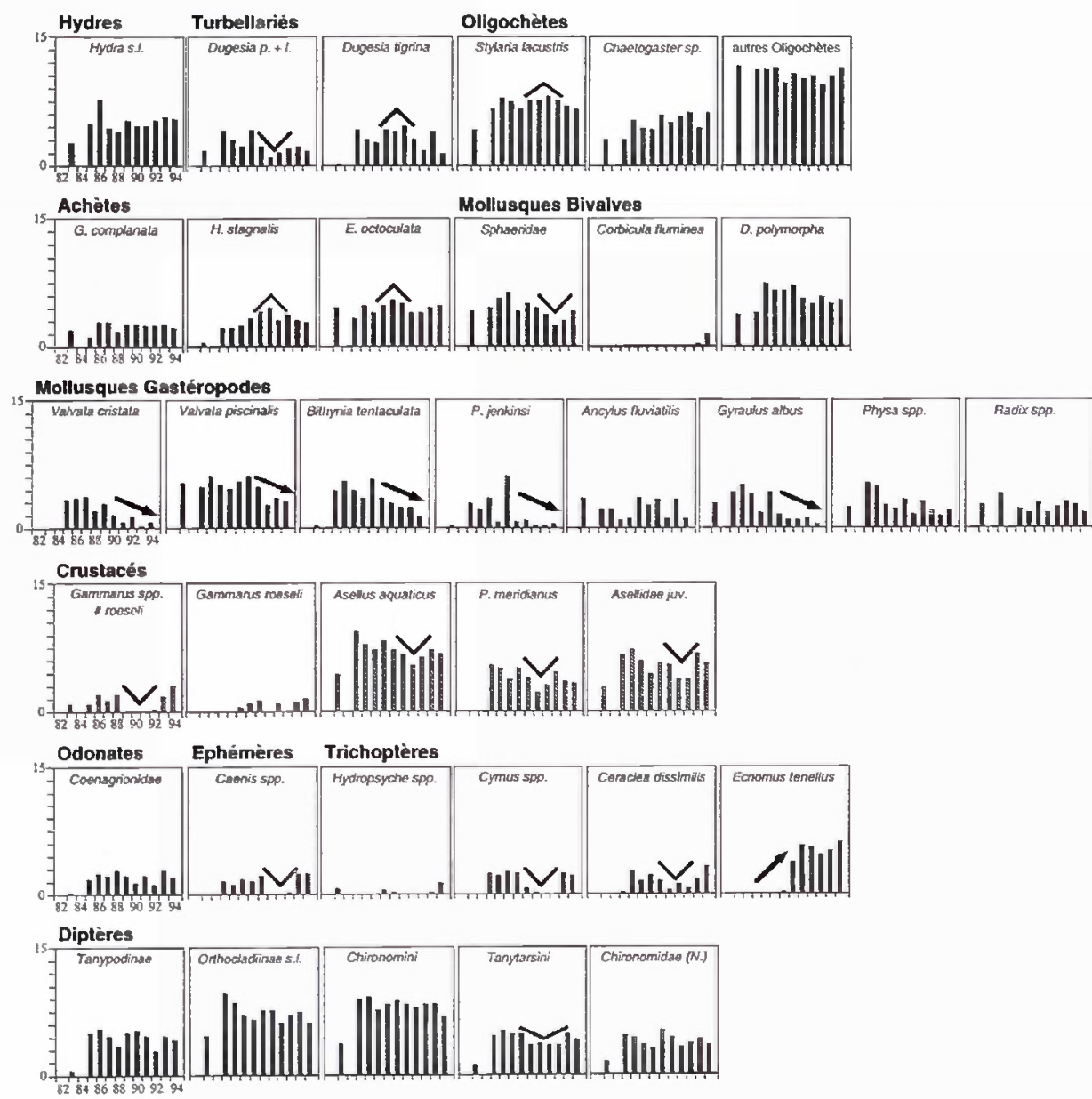

Fig. 11. - Evolution inter-annuelle de l'abondance moyenne par prélévement (nombre d'individus exprimé en $\log _{2}(x+1)$ ) des taxons les plus significatifs (abondance totale $-0,1 \%$, hormis Corbicula fluminea). Les flèches et les traits indiquent différents tendances évolutives.

Fig. 11. - Yearly evolution of the average abundance per sample (number of individuals expressed as $\log _{2}(x+1)$ ) of the most significant macroinvertebrates (total abundance $-0.1 \%$, except for Corbicula fluminea). Arrows and lines indicate different evolution tendancies.

avec les variations de débit est un phénomène important dans le fonctionnement écologique du fleuve. Toutefois, combiné à l'aménagement du fleuve qui a créé des conditions propices (diminution de la vitesse du courant, réchauffement, batelierie), cela a également favorisé des processus migratoires et/ou invasifs (cas des Crustacés Crangonyx pseudogracilis, Gammarus roeseli et Gammarus tigrinus et des Mollusques Lithoglyphus 
naticoïdes et Corbicula fluminea, en particulier), vis-à-vis desquels il est nécessaire d'être particulièrement vigilant: des phénomènes de compétition spatiale et trophique entre les nouveaux immigrants et les espèces autochtones ont en effet été observés sur le Rhin et la Moselle (Van der Velde et al., 1994; Bachmann et al., 1997). Ainsi, Corbicula fluminea, rencontrée à Péage-de-Roussillon à partir de 1992 sur l'axe principal du fleuve, est depuis en accroissement constant, tant du point de vue spatial qu'en termes d'effectifs.

\subsection{L'ichtyofaune}

Parmi les 56 espèces de Poissons recensées dans le bassin du Rhône (Changeux, 1994), 32 espèces appartenant à 11 familles ont été échantillonnées dans le secteur de Péage-de-Roussillon entre 1985 et 1994. A titre de comparaison, 35 espèces (dont 31 ont été retrouvées à Péage-de-Roussillon) ont été capturées sur le Haut-Rhône (Persat et al., 1995) et 34 au niveau du site de Cruas-Meysse (Carrel et al., 1995; CEMAGREF, 1995). La composition spécifique du peuplement est donc bien représentative de la partie médiane du Rhône, montrant une absence de certaines espèces très exigeantes vis-à-vis de la qualité de l'eau et de l'habitat comme la Lotte ou la Vandoise, encore présentes dans certains secteurs du HautRhône, d'une part; de la plupart des grands migrateurs (Alose, Lamproie) et des espèces méditerranéennes (Toxostome, Apron), d'autre part.

Le classement des espèces suivant les valeurs décroissantes de leur abondance relative montre que le Gardon représente $40 \%$ des effectifs échantillonnés, viennent ensuite le Chevaine, l'Ablette, la Perche-soleil et la Brème bordelière (fig. 12). Ces cinq espèces représentent $88 \%$ des effectifs capturés au cours des dix années d'échantillonnage. Lorsque l'on considère l'occurrence des espèces et non plus leur abondance, la distribution de fréquences des valeurs observées est plus équilibrée. Le Chevaine, le Gardon et la Perche-soleil montrent un pourcentage d'occurrence variant entre 16 et $22 \%$, les valeurs calculées pour l'Ablette et la Brème bordelière varient entre 6 et $7 \%$. La décroissance des valeurs est ensuite beaucoup plus régulière que lorsque l'on s'intéresse à l'abondance des espèces. Malgré tout, le peuplement apparait comme étant globalement très déséquilibré.

L'altération de l'habitat induite par les divers aménagements (endiguement et construction d'aménagements hydroélectriques) et la dégradation de la qualité physico-chimique de l'eau, par rapport au HautRhône, ont conduit à la disparition des populations les plus exigeantes vis-à-vis des facteurs fondamentaux comme la température de l'eau et l'oxygène dissous. Le peuplement actuellement en place est un peuple- 


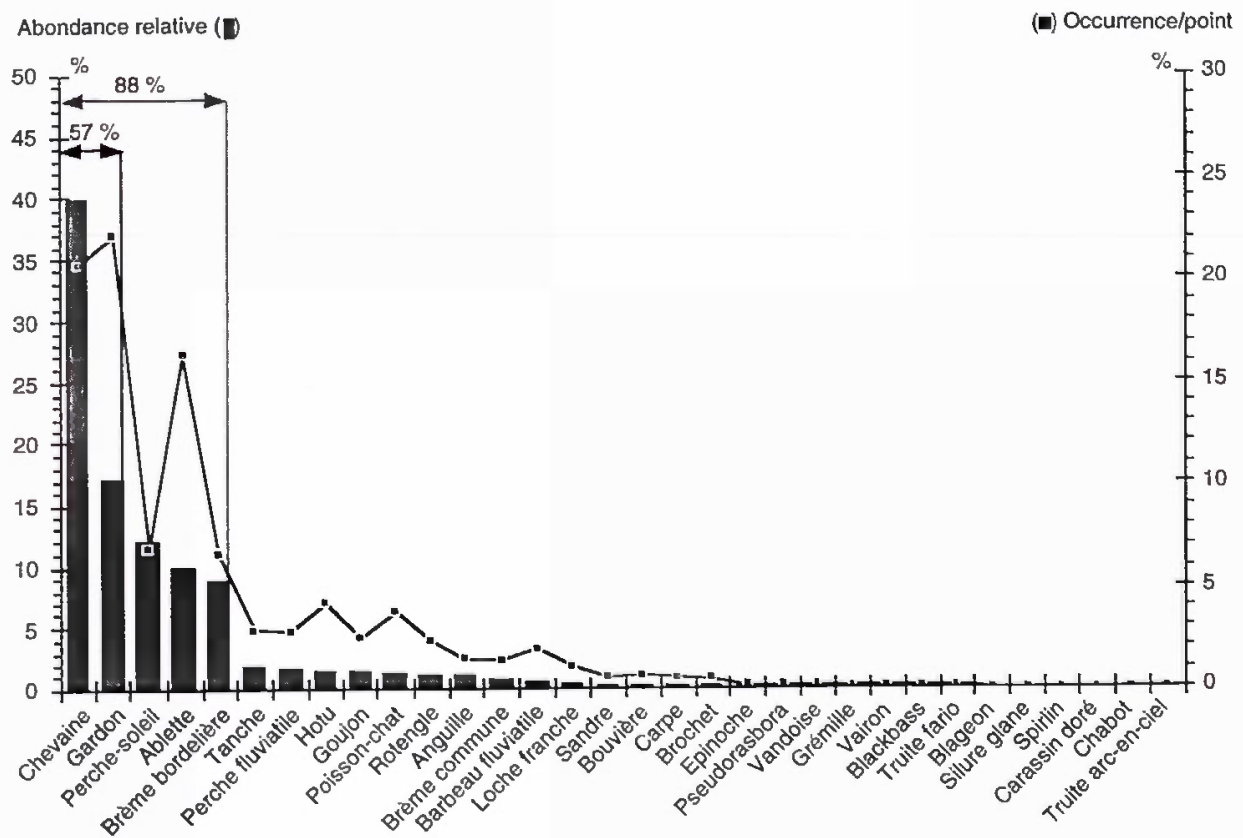

Fig. 12. - Abondance relative et occurrence des différentes espèces de poissons échantillonnées entre 1985 et 1994.

Fig. 12. - Relative abundance and occurrence of fish species sampled between 1985 and 1994.

ment relictuel dominé par des espèces euryèces qui trouvent dans ce milieu les conditions suffisantes pour accomplir leur cycle vital. L'examen des histogrammes en classes de tailles des espèces dominantes montrent que le recrutement s'est effectué normalement pour ces espèces durant la période étudiée (ARALEPBP, 1997).

L'analyse synthétique de la distribution des critères richesse spécifique, abondance moyenne et occurrence moyenne par station et par saison (fig. 13) montre que pour ces trois descripteurs les valeurs les plus élevées sont observées au prin- temps et en été, et surtout dans les stations 3,4 et 5 . Ces observations reflètent le rythme d'activité des poissons. Ceci met donc en évidence des différences inter-stations et inter-saisons qui doivent être analysées plus précisément.

L'analyse des variations de structures spatio-temporelles par l'étude des occurrences par pêche (AFC décentrée globale non présentée ici) montre que les variations enregistrées sont dues en priorité au succès de la reproduction des espèces dominantes et qu'il existe une interaction entre les effets stationnels et saisonniers. Les faibles pourcentages d'iner- 


$\begin{array}{ccccc}\text { Station 1 } & \text { Station } 2 & \text { Station } 3 & \text { Station } 4 & \text { Station } 5 \\ \text { Retenue amont } & \text { Retenue aval } & \text { Canal de fuite } & \text { Rhône court-circuité } & \text { Plan d'eau }\end{array}$

A - Richesse spécifique moyenne par station et par saison

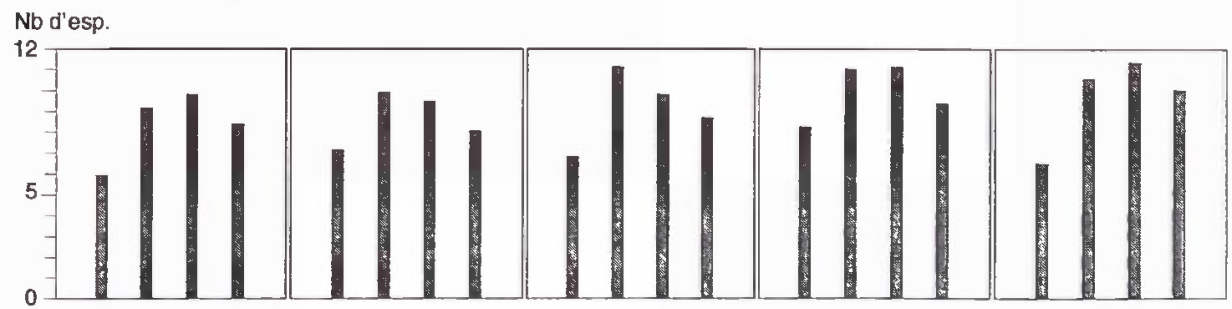

B - Abondance moyenne par station el par saison

Nb d'indiv,

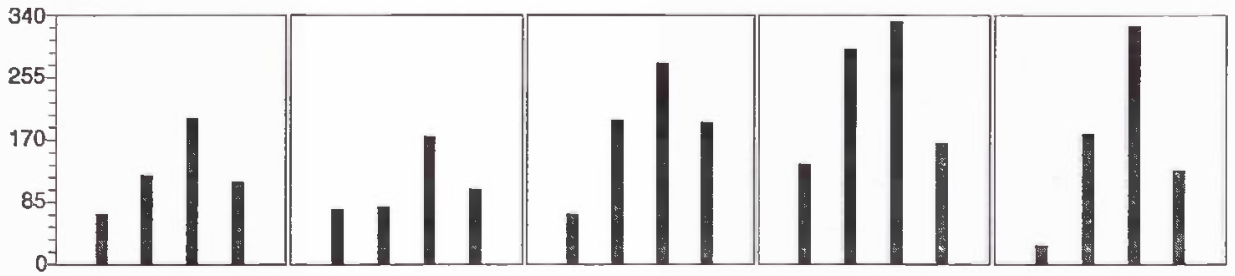

C-Occurrence moyenne par station el par saison

$\%$ EPA non vides

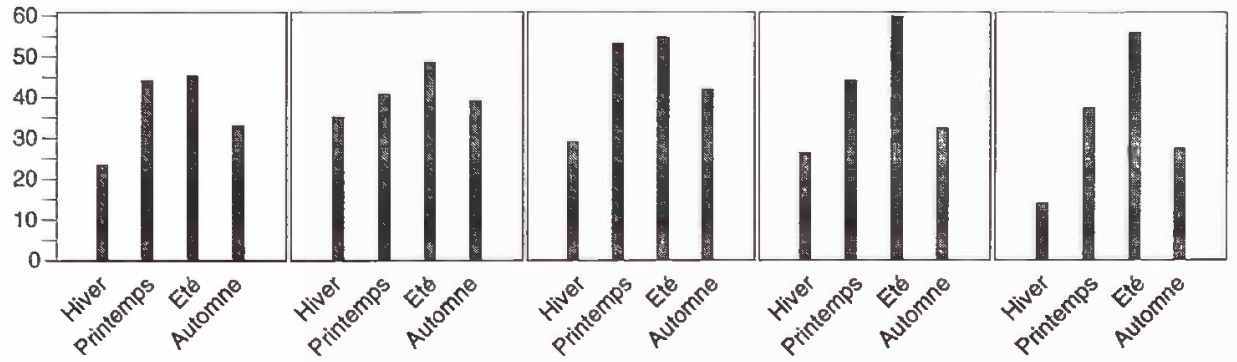

Fig. 13. - Structure du peuplement piscicole : richesse spécifique, abondance et occurrence moyennes par station et par saison.

Fig. 13. - Structure of the fish fauna : average richness, average abundance and average occurrence per station and per season.

tie associés aux premiers axes de l'AFC décentrée ( $28 \%$ pour les 2 premiers axes) soulignent la faible structure générale du tableau de données, c'est-à-dire qu'il n'existe pas de différences majeures de composition faunistique entre les différentes pêches, que ces différences soient d'ordre stationnel ou temporel. II devient alors beaucoup plus pertinent de rechercher l'évolution de chaque facteur (analyses inter- et intra-classes), par exemple rechercher l'évolution temporelle de la composition faunistique de 
chaque station. En raison du caractère redondant des interprétations des analyses intra-classes d'une part et de l'interaction des deux effets saisonnier et stationnels d'autre part, seuls les résultats d'une analyse intra-classes couplant ces deux critères (stations + saisons) seront interprétés en détail.

Les trois premiers axes de cette analyse prennent en compte $30 \%$ de l'inertie intra-classes, attestant de la répartition de l'information sur un grand nombre d'axes factoriels. La figure 14B montre que la variabilité intra stationnelle dans le plan $\mathrm{F} 1 \times \mathrm{F} 2$ de l'analyse est principalement marquée pour la saison estivale. Quelque soit la station, et de manière particulièrement nette au niveau des stations 1 et 2 (amont et aval immédiat de la centrale), cette variabilité est principalement marquée pour l'année 1989 , et dans une moindre mesure 1994, pour l'axe 1 et pour l'année 1987, voire 1990 et 1992, pour l'axe 2. La distribution des espèces dans le plan F1XF2 de l'analyse montre la rareté des juvéniles (première classe de taille pour la plupart des espèces) dans les pêches d'été 1987 principa- lement caractérisées par des poissons de grande taille (Ablette abl2 et 3 , Brème bordelière brb3 et 4 , Gardon gar3, Hotu hot3, etc.) et la forte occurrence de ces juvéniles en été 1989 (brb1, gar1, abl1, etc.) (fig. 14C). La proportion très importante de juvéniles dans les échantillons collectés en 1989 donne un caractère exceptionnel à cette année mais a très probablement eu une forte influence sur le succès de la reproduction en 1990 , les poissons nés en 1990 se trouvant en concurrence directe avec ceux de la cohorte 1989, ce qui peut expliquer la rareté des juvéniles de l'année en été 1990. La structure globale du peuplement de poissons dans le secteur étudié dépend donc principalement des facteurs hydroclimatiques, ceux-ci influençant le succès ou non de la reproduction (cf le déficit hydrologique et la température importante de l'année 1989 dans le premier cas, l'excédent hydrologique dans le second cas).

La projection des pêches dans le plan $\mathrm{F} 1 \times \mathrm{F} 3$ de l'analyse montre que la différence entre les stations 1 et 2 est principalement due à la présence de poissons-chats en automne 1986 et en hiver 1987 dans la station située

Fig. 14. - Evolution de la faune piscicole. Analyse Factorielle des Correspondances Décentrée intra stations-saisons, plan F1xF2: graphe des valeurs propres $(A)$, carte factorielle des relevés décomposée par station et par saison (B), carte factorielle des taxons dominants (15) découpés en classes de tailles (C).

Fig. 14. - Evolution of fish communities. Within-stations-seasons Decentered Correspondance Analysis, plane $\mathrm{F} 1 \times \mathrm{F} 2$ : eigenvalues graph (A), factorial map of the samples decomposed per station and per season (B), factorial map of dominant species (15) separated in size classes (C). 

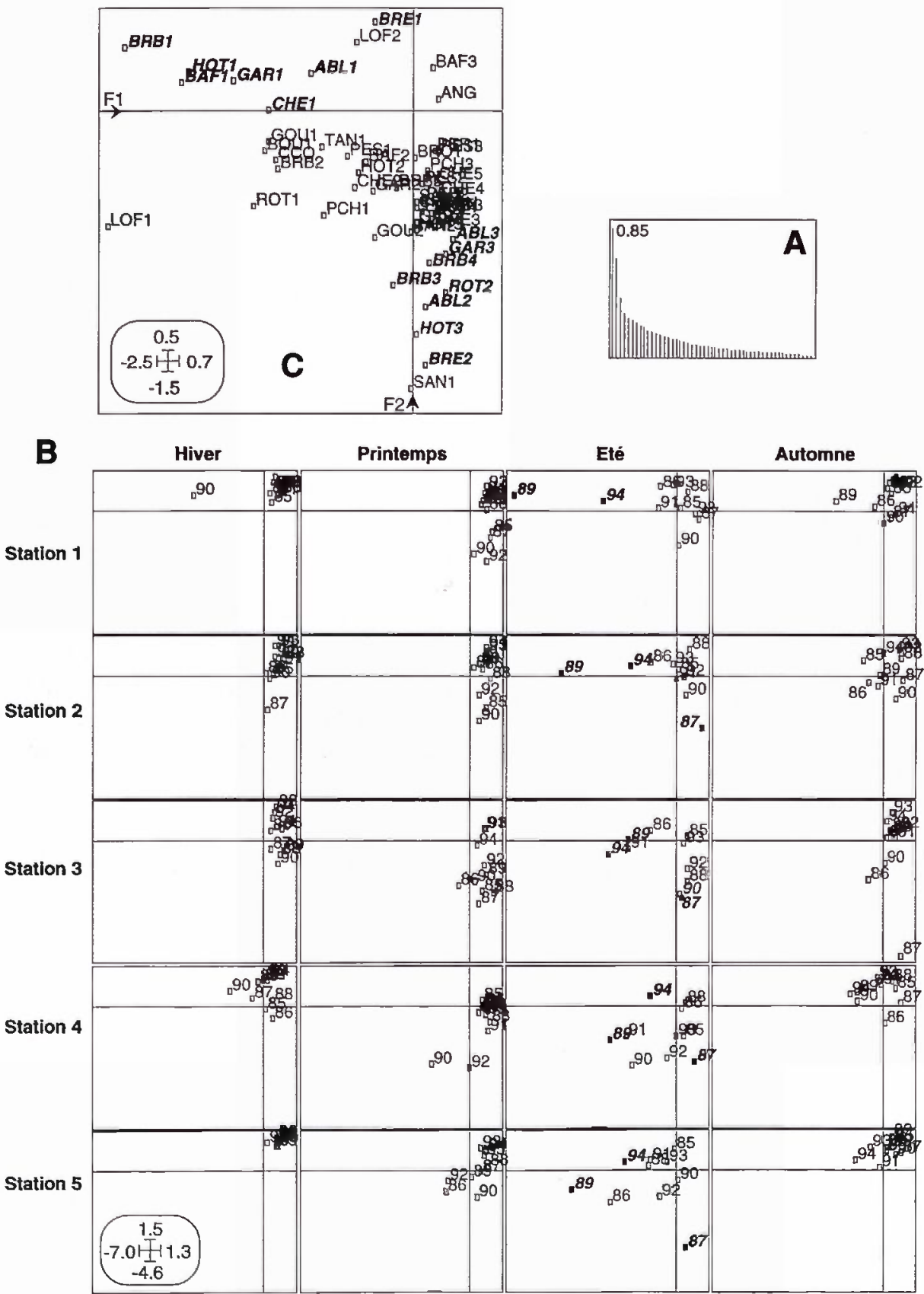

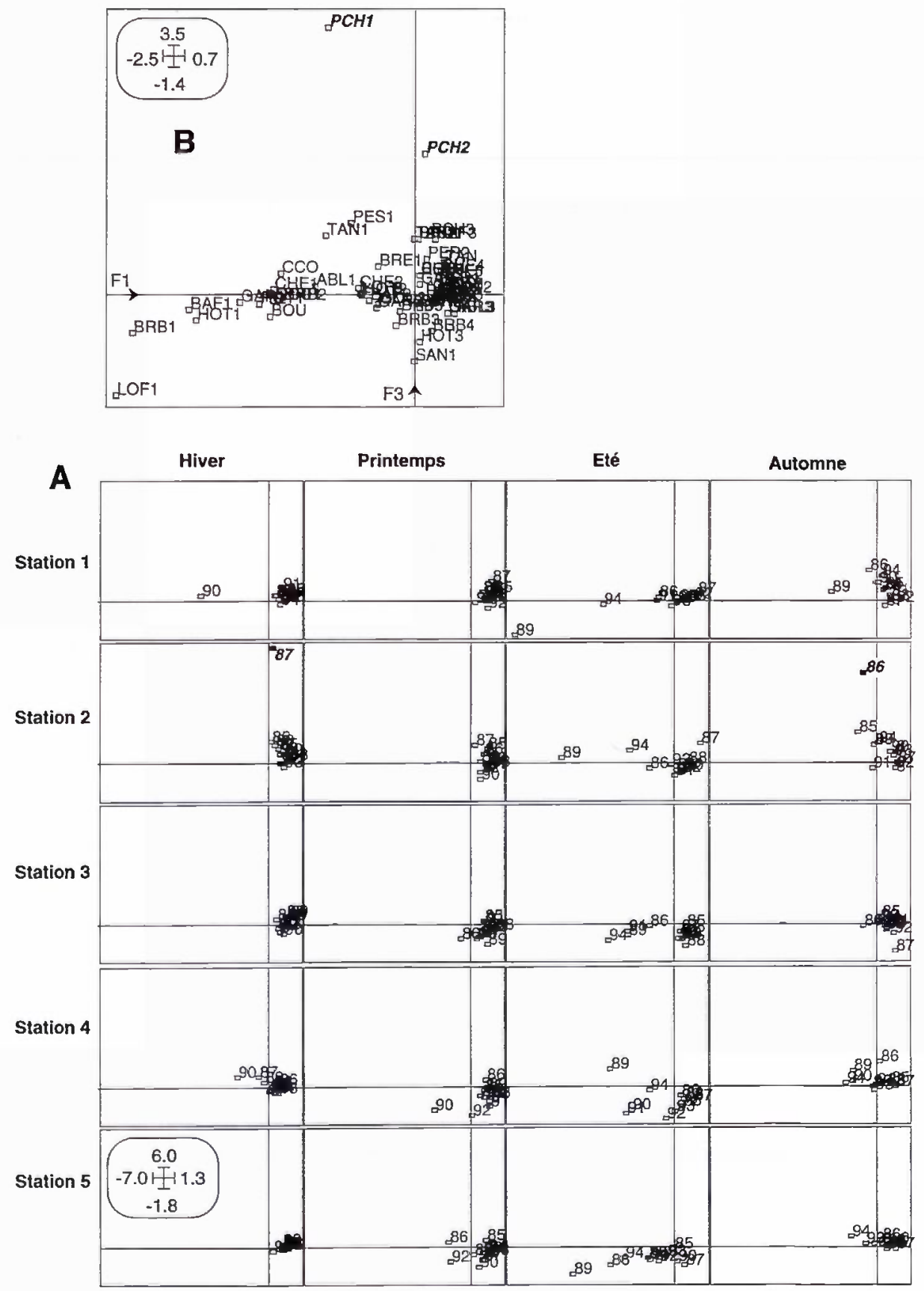
à l'aval de la centrale (fig. 15A et 15B), le rejet d'eau chaude pouvant constituer un facteur attractif en hiver pour cette espèce thermophile.

Cette synthèse des données piscicoles collectées sur dix ans montre ainsi l'intérêt des études à long terme dans le cas de systèmes complexes comme les écosystèmes fluviaux. En effet, la majorité des espèces qui constituent les ichtyocénoses des cours d'eau de grande dimension sont des espèces longévives dont les traits d'histoire de vie (fécondités, taille des œufs, etc.) en font des espèces adaptées aux variations hydrologiques naturelles. Les études à long terme constituent donc le meilleur moyen de séparer les événements à caractère ponctuel, tel que l'effet d'une crue, des événements dont les effets sont encore sensibles sur le long terme, tel que le recrutement durable chez une espèce particulière.

\section{DISCUSSION. INFLUENCE DU REJET THERMIQUE}

L'examen des descripteurs pris individuellement indique que la dynamique physico-chimique et biologique du secteur est sous la dépendance des conditions hydroclimatiques qui déterminent les variations saisonnières et inter-annuelles de l'hydrologie et de la température. Quel que soit le descripteur considéré, le positionnement des deux stations situées à l'amont et l'aval du site (stations 1 et 2 sur la retenue) est toujours très proche sur les diverses cartes factorielles issues des analyses inter-stations (fig. 16), attestant de compositions physico-chimiques et biologiques très voisines. Cet ensemble est lui-même distinct des stations 3 et 4 d'une part (stations les plus courantes), de la station 5 d'autre part (plan d'eau individualisé), en particulier sur le plan biologique (fig. 16B à 16E).

Linfluence du rejet d'eau réchauffée se manifeste par une augmentation de la température de l'eau à l'aval du site, et dans une moindre mesure dans la partie court-circuitée, où un relèvement des minima journaliers est constaté lorsque la centrale fonctionne à pleine puissance, mais les effets sur la faune et la flore sont modérés, voire non significatifs. Ils sont probablement masqués par les modifications anthropiques antérieures à l'implantation de la centrale : aménagement CNR, rejets domestiques et industriels divers. Ainsi, si le rejet d'eau chaude peut constituer un fac-

Fig. 15. - Evolution de la faune piscicole. Analyse Factorielle des Correspondances Décentrée intra stations-saisons, plan $\mathrm{F} 1 \times \mathrm{F} 3$ : carte factorielle des relevés décomposée par station et par saison (A), carte factorielle des taxons dominants (15) découpés en classes de tailles (B).

Fig. 15. - Evolution of fish communities. Within-stations-seasons Decentered Correspondance Analysis, plane F1xF3 : factorial map of the samples decomposed per station and per season (A), factorial map of dominant species (15) separated in size classes (B). 

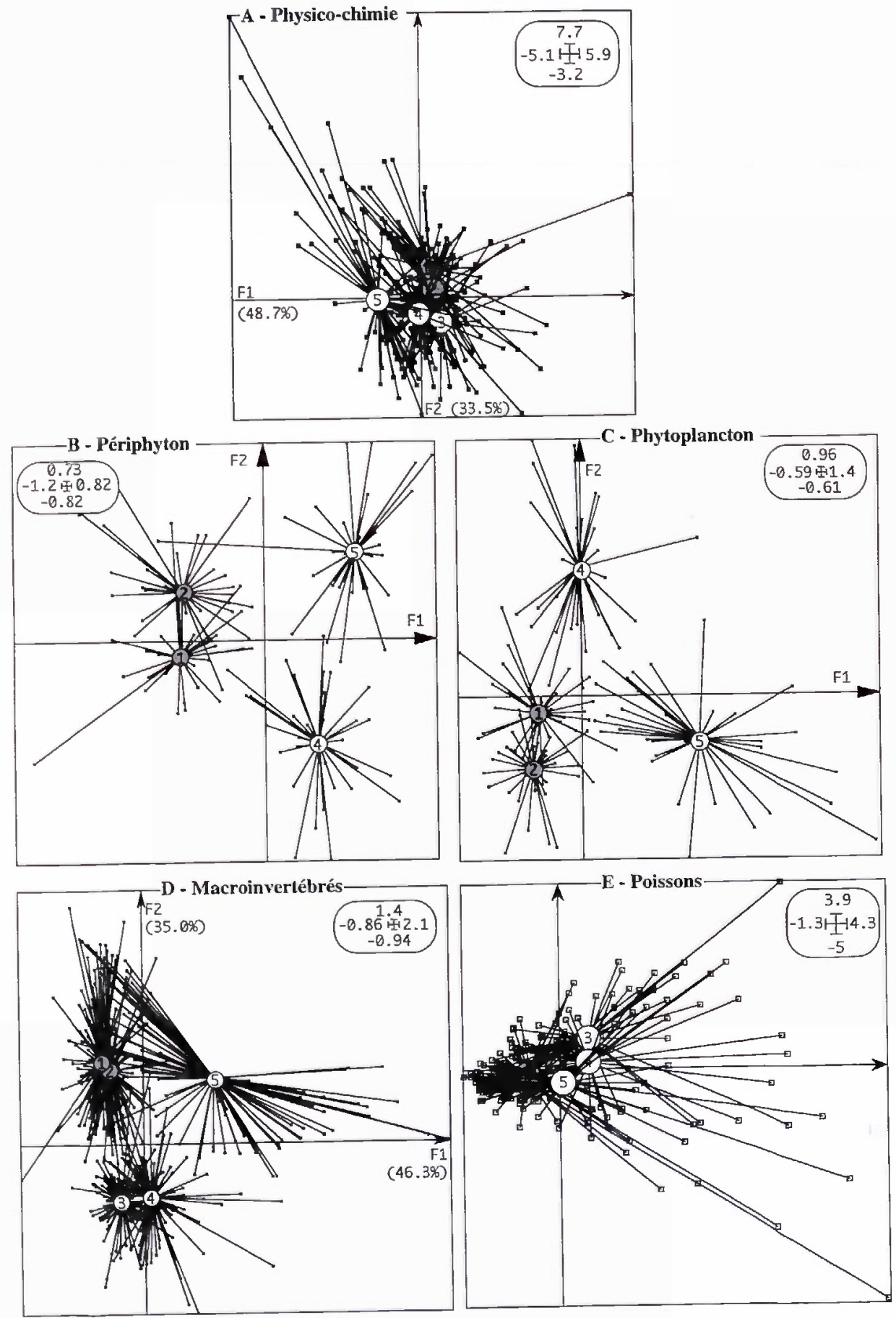
teur attractif en hiver pour des espèces thermophiles (Poissons-chats, quelques algues périphytiques), à l'inverse, les variations inter-annuelles des espèces végétales dominantes, ou d'espèces plus particulières (Phragmites australis, Ranunculus fluitans), traduisent l'intervention de phénomènes (hydrologie, sédimentation, eutrophisation) qui ne sont pas directement liés à la mise en service puis au fonctionnement de la centrale. De la même façon, le degré d'affinité réciproque entre les peuplements de macroinvertébrés de ces deux stations, exprimé par l'indice de Jaccard, révéle une similarité entre l'amont et l'aval de la centrale, tous types de prélèvements et dates confondus, de près de $63 \%$ (ARALEPBP, 1997).

\section{CONCLUSION}

Les caractéristiques physiques, chimiques et biologiques du BasRhône en général, et du RhôneMoyen en particulier, sont marquées, en premier lieu, par l'anthropisation du fleuve et de sa vallée (Fruget, 1992). Ainsi, d'un point de vue biologique, le système a dérivé vers un type potamique lent, composé d'espèces ubiquistes et résistantes et de quelques espèces plus sensibles mais relictuelles. D'un point de vue physico-chimique, ses grandes dimensions et l'inertie que cela confère à la masse d'eau vis-à-vis des apports extérieurs permettent au milieu d'assimiler des charges minérale et organique importantes.

L'hydrologie et la température apparaissent alors comme les événements majeurs régissant la dynamique physico-chimique et biologique du secteur de Péage-de-Roussillon: variations inter-annuelles des paramètres physico-chimiques, de la richesse, de la diversité et de l'abondance des différentes espèces végétales et animales. La saisonnalité des facteurs thermiques et hydrologiques et les conditions hydroclimatiques sont les principaux facteurs de contrôle. Ceci se vérifie quelque soit le descripteur considéré. Ainsi, le rôle charnière joué par l'année 1989 hydrologiquement déficitaire et thermiquement "excédentaire" a été mis en évidence. En second lieu, la dynamique des apports nutritifs gouverne également l'évolution de certains peuplements (cf en particulier le périphyton).

Dans ce cadre général, l'impact du rejet d'eau réchauffée de la centrale s'avère difficile à apprécier. Ce constat n'apparait toutefois pas propre au seul site de Saint-Alban, mais est général à l'ensemble des aménagements électro-nucléaires du Rhône, notamment aux sites de Bugey et de Cruas (Roger et al., 1991; CEMAGREF, 1995), voire à d'autres sites

Fig. 16. - Comparaison des analyses inter-stations réalisées sur les différents descripteurs.

Fig. 16. - Comparison of between-stations analyses done on the different descriptors. 
tels que ceux de Fessenheim sur le Rhin (Pierre, 1996) ou de Dampierre sur la Loire (Lair et al., 1996).

Cette influence se traduit essentiellement par une augmentation de la température de l'eau à l'aval du site, voire dans la partie court-circuitée, mais dont les effets sur la faune et la flore sont beaucoup plus ténus. II faut malgré tout se montrer vigilant afin que celle-ci, venant en complément d'autres sources d'altération des caractéristiques écologiques du fleuve (rejets chimiques, aménagements physiques, pollutions accidentelles,...), ne les amplifie pas à plus ou moins longue échéance. Ceci est particulièrement vrai pour la partie courtcircuitée dont le peuplement, bien qu'altéré et dominé par des espèces faunistiques tolérantes et à large spectre, reste encore diversifié avec la présence de différents taxons invertébrés et piscicoles plus potamiques et rhéophiles (Trichoptères Hydropsychidés, Barbeau fluviatile, par exemple), mettant en lumière l'importance des conditions locales : hétérogénéité de l'habitat par rapport à la retenue, présence d'un radier à l'amont.

Dans ce cadre, l'étude de l'évolution des hydrosystèmes fluviaux et de la dynamique de leurs peuplements impliquent une continuité d'observation et de récolte de données que seule permet une surveillance à long terme, nécessitant la mise en place de véritables observatoires écologiques du milieu naturel, tels que les sites du programme LTER (Magnuson, 1990). Cet auteur met également en garde sur les risques d'interprétations erronées de phénomènes observés trop ponctuellement, pour lesquels il est difficile de distinguer les perturbations anthropiques des fluctuations naturelles à moyen ou long terme (Schindler, 1987). De la même façon, Persat (1991) considère que le contexte spatio-temporel conventionnel dans lequel s'inscrit les études en milieu fluvial est fondamentalement trop étroit. Celles-ci exigent de disposer d'informations sur l'ensemble des composantes de l'hydrosystème considéré. Or, l'obtention de telles informations nécessite un suivi continu dans l'espace et dans le temps. On retrouve ainsi une des principales préoccupations de la recherche en écologie (Likens, 1989; Wiens et al., 1986).

L'intérêt essentiel de telles recherches tient, pour une large part, à la méthodologie utilisée et à la stratégie d'échantillonnage dans l'espace et dans le temps choisie. L'aspect répétitif de cette dernière permet ainsi de présenter un fonctionnement dynamique des systèmes étudiés et d'en tirer des leçons dans le domaine de l'écologie appliquée (Bellan \& Bourcier, 1990; Reish \& Bellan, 1995). En retour, cette approche appliquée des problèmes environnementaux contribue grandement à une meilleure connaissance du fonctionnement des hydrosystèmes fluviaux. 


\section{REMERCIEMENTS}

Cette étude est financée par EDF dans le cadre du suivi hydrobiologique du Rhône au niveau du CNPE de Saint-Alban - St-Maurice. Les auteurs remercient vivement Mr Etienne Coatleven (EDF Saint-Alban) pour l'intérêt manifesté envers ce suivi et sa collaboration pour la fourniture des données concernant le fonctionnement de la centrale et les enregistrements thermiques.

Ce suivi nécessite également la contribution de nombreux collègues, tant pour les prélèvements sur le terrain que pour leur dépouillement au laboratoire. Qu'ils trouvent ici l'expression de nos chaleureux remerciements.

\section{REFERENCES}

AERMC 1997. Indice Biologique Global Adapté aux grands cours d'eau et rivieres profondes (I.B.G.A.). Protocole expérimental. Rapport Agence de l'Eau Rhône-Méditerranée-Corse, $45 \mathrm{p}$.

ARALEPBP 1997. Suivi hydrobiologique du Rhône au niveau de la centrale nucléaire de Saint-Alban - St-Maurice. Synthèse décennale 1985-1994. Rapport à EDF, $94 \mathrm{p}$.

ARALEPBP 1983. Etat de référence hydrobiologique du Rhône au niveau de l'aménagement CNR de Péage-deRoussillon avant la mise en service de la centrale nucléaire de St-Mauricel'Exil. Rapport à EDF, 75 p.

Bachmann V., Usseglio-Polatera P., Cegielka E., Wagner P., Poinsaint J.F. \& Moreteau J.C. 1997. Premières observations sur la coexistence de Dreissena polymorpha, Corophium curvispinum et Corbicula spp. dans la rivière Moselle. Bull. Fr. Pêche Piscic. 344/345, 373-384.

Balocco-Castella C. 1988. Les macrophytes aquatiques des milieux abandonnés par le Haut-Rhône et l'Ain: diagnostic phyto-écologique sur l'évolution et le fonctionnement de ces écosystèmes. Thèse de Doctorat, Univ. Lyon I, 150 p.

Bellan G. \& Bourcier M. 1990. Les enseignements d'une étude sur dix ans (1976-1986) des peuplements au large d'un émissaire d'eaux usées: Marseille-Cortiou. Cah. Biol. Mar. 31, 225-249.

Bournaud M. \& Amoros C. 1984. Des indicateurs biologiques aux descripteurs de fonctionnement: quelques exemples dans un système fluvial. Bull. Ecol. 15 (1), 57-66.

CEMAGREF 1995. Suivi hydrobiologique du Rhône. Site de Cruas-Meysse. 1982-1995. Rapport à EDF, 45 p.

Carrel G., Pont D. \& Rivier B. 1995. Variabilité temporelle des peuplements piscicoles dans la section médiane du Bas-Rhône. Bull. Fr. Pêche Piscic. 337/338/339, 101-111.

Changeux T. 1994. Structure des peuplements de poissons à l'échelle du bas$\sin$ Rhodanien. Approche régionale et organisation longitudinale, exploitation des captures pêche aux engins. Thèse de Doctorat, Univ. Lyon 1, 214 p.

Chessel D. \& Hanafi M. 1996. Analyse de la co-inertie de $\mathrm{k}$ nuages de points. Rev. Statistique Appliquée XLIV (2), 35-60.

Connell J.H. 1978. Diversity in tropical rain forests and coral reefs. Science 199 , 1302-1310.

Crouzet P. \& Amezal A. 1982. Méthodologie d'analyse des causes et remèdes à l'eutrophisation des cours d'eau. Mise au point sur le bassin de l'indre (France). Rapport XVIIe Journées Hydraul. Soc. Hydrotechn. Fr., 7 p. 
Del Giorgio P.A., Vinocur A.L., Lombardo R.J. \& Tell H.G. 1991. Progressive changes in the structure and dynamics of the phytoplankton community along a pollution gradient in a lowland river - a multivariate approach. Hydrobiologia 224, 129-154.

Descy J.P. 1993. Ecology of the phytoplankton of the River Moselle : effects of disturbances on community structure and diversity. Hydrobiologia 249, 111-116.

Descy J.P. 1992. Eutrophication in the River Meuse. In Sutcliffe D.W. \& Jones J.G. (Eds), Eutrophication: research and application to water supply, Freshwater Biological Association, The Ferry House, Ambleside (G.B.), 132-142.

Dolédec S. \& Chessel D. 1989. Rythmes saisonniers et composantes stationnelles en milieu aquatique. II. Prise en compte et élimination d'effets dans un tableau faunistique. Acta Oecol., Oecol. Gener. 10 (3), 207-232.

Dolédec S., Chessel D. \& Olivier J.M. 1995. L'analyse des correspondances décentrée: application aux peuplements ichtyologiques du Haut-Rhône. Bull. Fr. Pêche et Piscic. 336, 29-40.

Dynesius M. \& Nilsson C. 1994. Fragmentation and flow regulation of river systems in the northern third of the world. Science 266, 753-762.

Fruget J.F. 1994. Invertebrates communities in a regulated floodplain of the Middle Rhône River. Verh. Internat. Verein. Limnol. 25 (3), 1549-1554.

Fruget J.F. 1992. Ecology of the Lower Rhône following 200 years of human influence: a review. Regulated Rivers 7 (3), 233-246.

Fruget J.F. 1991. The impact of river regulation on the lotic macroinvertebrate communities of the Lower Rhône, France. Regulated Rivers 6 (4), 241-255.

Fruget J.F. \& Michelot J.L. 1997. Dérives écologiques et gestion du milieu fluvial rhodanien. Rev. Géogr. Lyon 72 (1), 35-48.

Khalanski M. \& Gras R. 1996. Rejets thermiques en rivières et hydrobiologie. Un aperçu sur l'expérience française. La Houille Blanche 5, 13-18.

Lair N. \& Sargos D. 1993. A 10 years study in the middle course of the River Loire. I - Patterns of change in hydrological, physical and chemical variables at the sites of the nuclear power plants, in relation to algal biomass. Hydroécol. Appl. 5 (1), 1-27.

Lair N., Sargos D. \& Reyes-Marchant P. 1996. Synthèse des études hydrobiologiques réalisées en Loire moyenne au niveau du site nucléaire de Dampierre-en-Burly (France). Hydroécol. Appl. 8 (1-2), 35-84.

Likens G.E. 1989. Long-term studies in ecology. Approches and alternatives. Springer, Berlin, $214 \mathrm{p}$.

Magnuson J.J. 1990. Long-term ecological research and the invisible present. BioScience 40 (7), 495-501.

Meurisse-Genin M., Reydams-Detollenaere A., Stroot P. \& J.C. Micha 1987. Les macroinvertébrés benthiques de la Meuse belge: bilan des cinq années de recherche (1980 à 1984). Arch. Hydrobiol. 109 (1), 67-88.

Michelot J.L. 1983. Evolution des paysages fluviaux de la Vallée du Rhône dans le secteur du Péage-de-Roussillon. Rev. Géogr. Lyon 58 (4), 307322.

Nelva A., Persat H. \& Chessel D. 1979. Une nouvelle méthode d'étude des prélévements ichtyologiques dans les grands cours d'eau par échantillonnage ponctuel d'abondance. C.R. Acad. Sci. Paris 289, 1295-1298.

Persat H. 1991. Espace et temps, pierres d'achoppement des études d'impact en milieu fluvial. Bull. Ecol. 22 (1), 203-212.

Persat H. \& Copp G.H. 1990. Electric fishing and point abundance sampling for the ichtyology of large rivers. In I.G. 
Cowx (Ed.), Developments in electric fishing" Fishing News Books, Blackwell, Cambridge, 27, 197-209.

Persat H. \& Olivier J.M. 1991. The point abundance sampling, a fishing strategy for large rivers : short presentation of the concept, its appliance and some results. In Penaz M. (Ed.), Biological monitoring of large rivers, Czechoslovak Academy of Sciences, Brno \& Slovak Academy of Sciences, Bratislava Publishers, 104-113.

Persat H., Olivier J.M. \& Bravard J.P. 1995. Stream and riparian management of large braided mid-European rivers, and consequences for fish. In N.B. Armantrout (ed.), Condition of the world's aquatic habitats, Proceedings of the World Fisheries Congress, Theme 1, Oxford \& IBH Publishing Co. Pvt. Ltd., New Delhi, 139-169.

Petts G.E., Möller H. \& Roux A.L. 1989. Historical change of large alluvial rivers in Western Europe. J. Wiley \& Sons (Eds), Chichester, 355 p.

Pierre J.F. 1996. Flore algale du Rhin canalisé : données récentes. Bull. Fr. Pêche Piscic. 341/342, 109-114.

Reish D.J. \& Bellan G. 1995. The longterm effects of municipal discharges from urban areas on the marine environment: a review. In D. Bellan, $\mathrm{G}$. Bonin \& C. Emig (Eds), Functioning and dynamics of natural and perturbed ecosystems, Tec \& Doc - Lavoisier, Paris, 701-743.

Roger M.C., Faessel B. \& Lafont M. 1991. Impact thermique des effluents du Cen- tre de Production Nucléaire du Bugey sur les invertébrés benthiques du Rhône. Hydroécol. Appl. 1 (3), 63-110.

Schindler D.W. 1987. Detecting ecosystem responses to anthropogenic stress. Can. J. Fish. Aquat. Sci. 44, 6-25.

Tachet H., Gaschignard-Fossati O., Cellot B. \& A. Berly 1988. Le macrobenthos de la Saône. Annls Limnol. 24 (1), 83100.

Thioulouse J., Dolédec S., Chessel D. \& Olivier J.M. 1995. ADE Software : multivariate analysis and graphical display of environmental data. In Guariso, G. \& Rizzoli, A. (Eds.), Software per l'ambiante, Pàtron Editore, Bologna, 5762.

Van der Velde G., Paffen B.G.P. \& Van den Brink F.W.B. 1994. Decline of zebra mussel populations in the Rhine: competition between two mass invaders (Dreissena polymorpha and $\mathrm{Co}$ rophium curvispinum). Naturwiss. 81 , 32-34.

Ward J.V. \& Stanford J.A. 1983. The intermediate-disturbance hypothesis: an explanation for biotic diversity patterns in lotic ecosystems. In Fontaine T.D. \& Bartell S.M. (Eds), Dynamics of lotic ecosystems, Ann Arbor Science Publ., Ann Arbor (USA), 347-356.

Wiens J.A., Addicott J.F., Case T.J. \& Diamond J. 1986. Overview: the importance of spatial and temporal scale in ecological investigations. In Diamond J. \& Case T.J. (Eds), Community ecology, Harper \& Row, New-York, 145153. 